\title{
AKARI observations of ice absorption bands towards edge-on young stellar objects
}

\author{
Y. Aikawa ${ }^{1}$, D. Kamuro ${ }^{1}$, I. Sakon ${ }^{2}$, Y. Itoh ${ }^{1}$, H. Terada ${ }^{3}$, J. A. Noble ${ }^{4}$, K. M. Pontoppidan ${ }^{5}$, \\ H. J. Fraser ${ }^{4}$, M. Tamura ${ }^{3}$, R. Kandori ${ }^{3}$, A. Kawamura ${ }^{3,6}$, and M. Ueno ${ }^{7}$ \\ ${ }^{1}$ Department. of Earth and Planetary Sciences, Kobe University, 657-8501 Kobe, Japan \\ e-mail: aikawa@kobe-u.ac.jp \\ 2 Department of Astronomy, School of Science, University of Tokyo, 7-3-1 Hongo, Bunkyo-ku, 113-0033 Tokyo, Japan \\ 3 National Astronomical Observatory of Japan, Osawa 2-21-1, Mitaka, 181-8588 Tokyo, Japan \\ ${ }^{4}$ Department of Physics, Scottish Universities Physics Alliance (SUPA), University of Strathclyde, Glasgow G4 ONG, UK \\ 5 Space Telescope Science Institute, 3700 San Martin Drive, Baltimore, MD 21218, USA \\ ${ }^{6}$ Department of Physics, Nagoya University, Furo-cho, Chikusa-ku, 464-8602 Nagoya, Japan \\ 7 Institute of Space and Astronautical Science, Japan Aerospace Exploration Agency, 3-1-1 Yoshinodai, Sagamihara, \\ 229-8510 Kanagawa, Japan
}

Received 26 October 2010 / Accepted 17 November 2011

\begin{abstract}
Context. Circumstellar disks and envelopes of low-mass young stellar objects (YSOs) contain significant amounts of ice. Such icy material will evolve to become volatile components of planetary systems, such as comets in our solar system.

Aims. To investigate the composition and evolution of circumstellar ice around low-mass young stellar objects (YSOs), we observed ice absorption bands in the near infrared (NIR) towards eight YSOs ranging from class 0 to class II, among which seven are associated with edge-on disks.

Methods. We performed slit-less spectroscopic observations using the grism mode of the InfraRed Camera (IRC) on board AKARI, which enables us to obtain full NIR spectra from $2.5 \mu \mathrm{m}$ to $5 \mu \mathrm{m}$, including the $\mathrm{CO}_{2}$ band and the blue wing of the $\mathrm{H}_{2} \mathrm{O}$ band, which are inaccessible from the ground. We developed procedures to carefully process the spectra of targets with nebulosity. The spectra were fitted with polynomial baselines to derive the absorption spectra. The molecular absorption bands were then fitted with the laboratory database of ice absorption bands, considering the instrumental line profile and the spectral resolution of the grism dispersion element.

Results. Towards the class 0-I sources (L1527, IRC-L1041-2, and IRAS 04302), absorption bands of $\mathrm{H}_{2} \mathrm{O}, \mathrm{CO}_{2}, \mathrm{CO}$, and XCN are clearly detected. Column density ratios of $\mathrm{CO}_{2}$ ice and $\mathrm{CO}$ ice relative to $\mathrm{H}_{2} \mathrm{O}$ ice are $21-28 \%$ and $13-46 \%$, respectively. If $\mathrm{XCN}$ is $\mathrm{OCN}^{-}$, its column density is as high as $2-6 \%$ relative to $\mathrm{H}_{2} \mathrm{O}$ ice. The $\mathrm{HDO}$ ice feature at $4.1 \mu \mathrm{m}$ is tentatively detected towards the class 0 -I sources and HV Tau. Non-detections of the $\mathrm{CH}$-stretching mode features around $3.5 \mu$ m provide upper limits to the $\mathrm{CH}_{3} \mathrm{OH}$ abundance of 26\% (L1527) and 42\% (IRAS 04302) relative to $\mathrm{H}_{2} \mathrm{O}$. We tentatively detect OCS ice absorption towards IRC-L1041-2. Towards class 0-I sources, the detected features should mostly originate in the cold envelope, while $\mathrm{CO}_{\text {gas }}$ and $\mathrm{OCN}^{-}$ could originate in the region close to the protostar, where there are warm temperatures and UV radiation. We detect $\mathrm{H}_{2} \mathrm{O}$ ice band towards ASR41 and 2MASSJ 1628137-243139, which are edge-on class II disks. We also detect $\mathrm{H}_{2} \mathrm{O}$ ice and $\mathrm{CO}_{2}$ ice towards $\mathrm{HV}$ Tau, HK Tau, and UY Aur, and tentatively detect CO gas features towards HK Tau and UY Aur.
\end{abstract}

Key words. circumstellar matter - infrared: ISM - stars: formation - astrochemistry

\section{Introduction}

In molecular clouds, protostellar envelopes, and protoplanetary disks, significant amounts of oxygen and carbon are in the form of molecules in ice mantles, such as $\mathrm{H}_{2} \mathrm{O}, \mathrm{CO}, \mathrm{CO}_{2}$, and $\mathrm{CH}_{3} \mathrm{OH}$ (e.g. Whittet 1993; Murakawa et al. 2000; Gibb et al. 2004). These interstellar ices are formed by the adsorption of gas-phase molecules onto grain surfaces and/or the grain-surface reactions of the adsorbed species (e.g. Aikawa et al. 2005).

Ices in circumstellar disks of low-mass young stellar objects are of special interest as they contribute to the raw material of planetary formation. Observations of disk ices, however, are not straightforward for the following two reasons, and thus remain limited in number. Firstly, ices exist mostly near the midplane at $r \geq$ several AU. Observations of disk ices are basically restricted to edge-on disks, where the ice bands absorb stellar light, scattered stellar light, and/or thermal emission of warm dust in the disk inner radius (Pontoppidan et al. 2005). The targets are naturally heavily extincted by dust, and thus are faint. Secondly, it is unclear whether the ice absorption bands, if detected, originate in the disk or other foreground components (i.e. ambient clouds). The source CRBR 2422.8-3423 was the first edge-on object towards which detailed ice observations were performed (Thi et al. 2002). Pontoppidan et al. (2005) concluded, via detailed analysis and modeling of the object, that only a limited amount $(<20 \%)$ of detected $\mathrm{CO}$ ice may exist in the disk, while up to $50 \%$ of water and $\mathrm{CO}_{2}$ may originate in the disk. They also found that the $6.85 \mu \mathrm{m}$ band, which is tentatively attributed to $\mathrm{NH}_{4}^{+}$, has a prominent red wing. Since this wing is reproduced by the thermal processing in the laboratory, it indicates that along the line of sight towards CRBR 2422.8-3423 there is warm ice in the disk.

Honda et al. (2009) succeeded in detecting water ice in a disk around a Herbig Ae star, which is not edge-on. They observed 
(a) 1152

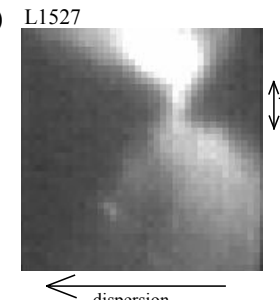

2MASSJ1628137-243139

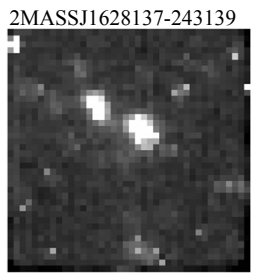

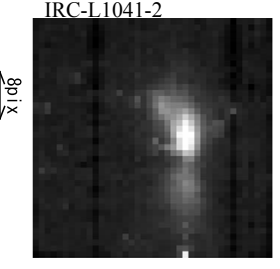

HV Tau

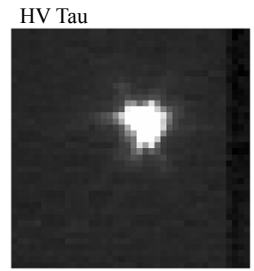

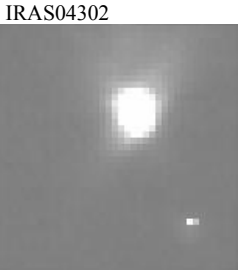

HK Tau
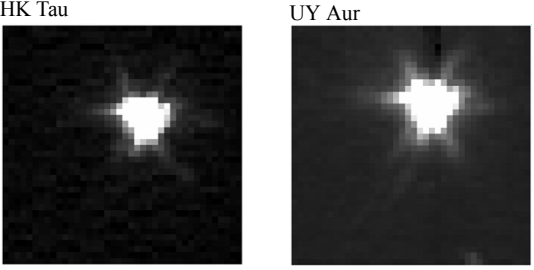

(b)
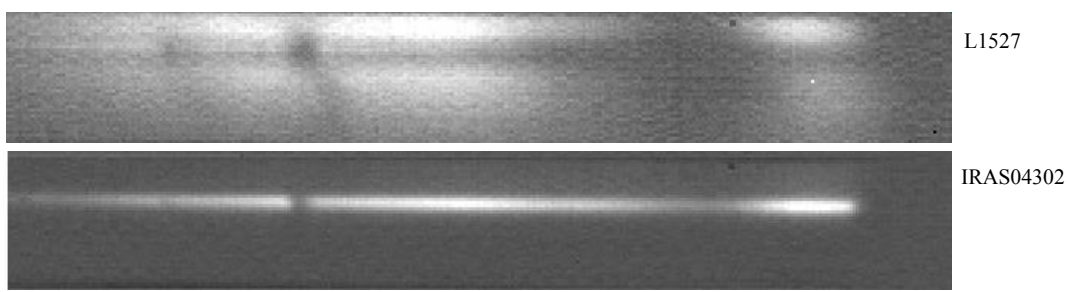

Fig. 1. a) Images of our targets taken by AKARI with the N3 filter (2.7-3.8 $\mu \mathrm{m})$. The stellar light is dispersed from right (short wavelength) to left (long wavelength). The FOV is $1^{\prime} \times 1^{\prime}$. The coordinate at the center of the FOV is listed in Table 1. Images of IRC-L1041-2 and 2MASS J1628137-243139 are damaged by cosmic rays, while images of HV Tau, HK Tau, ASR41, and UY Aur are affected by saturation and column pull down. b) Spectral images of 1527 and IRAS 04302.

scattered light from a disk around HD142527 at multiple wavelength bands around $\sim 3 \mu \mathrm{m}$. This method can be a powerful tool for investigating the spatial distribution of ices in disks. However, it would currently be difficult to detect ice features other than the $3 \mu \mathrm{m}$ water band via scattered light.

In the present paper, we report near infrared (NIR) (2.5-5 $\mu \mathrm{m})$ observations of edge-on disks using the grism mode of the IRC on board AKARI. We selected seven edge-on YSOs; three are considered to be in a transient state of class 0 -I, and four are class II (see Sect. 2 for more detailed descriptions). Although the absorption by the protostellar envelope may overwhelm the absorption by the disk in class 0-I YSOs, envelope material along our line of sight (perpendicular to the rotation axis and outflow) is likely to end up in the midplane region of the forming disk, and ices with a sublimation temperature of $>50 \mathrm{~K}$ (i.e. $\mathrm{CO}_{2}$ and $\mathrm{H}_{2} \mathrm{O}$ ) would survive the accretion shock onto the disk (Visser et al. 2009).

Ice observations towards low-mass YSOs have been performed using Spitzer Space Telescope (SST) and various ground-based telescopes such as the Very Large Telescope (VLT) and Subaru. Pontoppidan et al. (2003a) observed the $4.67 \mu \mathrm{m}$ CO ice band, which is observable from the ground, towards 39 YSOs. Using SST, Furlan et al. (2008) and Pontoppidan et al. (2008) observed the strong $\mathrm{CO}_{2}$ ice band at $15.3 \mu \mathrm{m}$ towards class I sources. Observations with SST also detected absorption bands of $\mathrm{CH}_{3} \mathrm{OH}, \mathrm{HCOOH}, \mathrm{H}_{2} \mathrm{CO}$, and $\mathrm{CH}_{4}$ (Boogert et al. 2008; Zasowski et al. 2009; Öberg et al. 2008). Since ground-based observations are restricted to atmospheric windows, and since SST is restricted to the mid infrared (5-36 $\mu \mathrm{m})$, NIR observations via AKARI are important to comprehend the full spectra of ices in circumstellar material. AKARI has a high enough sensitivity for the spectroscopic observation of low-mass YSOs; the sensitivity of the AKARI grism mode at $3 \mu \mathrm{m}$ is $120 \mu \mathrm{Jy}$ ( $1 \sigma, 10 \mathrm{~min})$, which is almost comparable to the $K$-band sensitivity of the spectroscopic observation at 8-m ground-based telescopes (VLT and Subaru). While the $L$-band sensitivity on the ground is $\sim 5$ mag or 100 times worse than at $K$-band, the sensitivity of the AKARI grism mode does not vary significantly at $2.5-4 \mu \mathrm{m}$, and only gradually worsens at longer wavelengths, by up to a factor of $3-4$ at $5 \mu \mathrm{m}$.

\section{Observations}

Observations were performed using the IRC (Onaka et al. 2007; Ohyama et al. 2007) on board the AKARI satellite (Murakami et al. 2007) from September 2006 to March 2007. We used the Astronomical Observational Template 04 (AOT04), designed for spectroscopy. The AOT04 replaces the imaging filters with transmission-type dispersers on the filter wheels to take nearand mid-infrared spectra. The field of view (FOV) consists of a $1^{\prime} \times 1^{\prime}$ aperture (covered by $40 \times 40$ pixels in the imaging mode) (Fig. 1a) and a $10^{\prime} \times 10^{\prime}$ aperture. The targets are set at the center of the $1^{\prime} \times 1^{\prime}$ aperture (without a slit), where only the NIR channel is available. The resolving power of the IRC grism mode is $0.00965 \mu \mathrm{m} /$ pixel, and the full-width at half maximum (FWHM) of the point spread function (PSF) is $\sim 2.9$ pixels in the NIR. The spectral resolution is thus $\sim 100$, which is sufficient to detect the ice absorption bands. In each observation, the following data are taken in a period of $600 \mathrm{~s}$ : two dark frames, one image frame with the N3 filter $(2.7-3.8 \mu \mathrm{m})$, and eight slitless spectral images.

Table 1 lists our targets, and Fig. 1a shows the N3 images of our targets. The stellar light is dispersed from right (short wavelengths) to left (long wavelengths). The position angle of the FOV is fixed; the dispersion direction is almost aligned with the ecliptic latitude. The nebulosity of our targets extends almost perpendicularly to the dispersion direction. As an example, the spectral images of L1527 and IRAS 04302 are shown in Fig. 1b. 
Table 1. Target list.

\begin{tabular}{lccc}
\hline \hline Name & Coordinates $(\mathrm{J} 2000)^{a}$ & Class & Note \\
\hline L1527(IRAS 04368+2557) & $04: 39: 53.6+26: 03: 05.5$ & 0-I & ${\text { WCCC }, i \sim 85^{\circ b}}^{c}$ \\
IRC-L1041-2 & $20: 37: 21,+57: 44: 13$ & 0-I & nearly edge-on \\
IRAS 04302+2247 & $04: 33: 16.45,+22: 53: 20.7$ & 0-I & $i \sim 90^{\circ} d$ \\
ASR 41 & $03: 28: 51.291,+31: 17: 39.79$ & II & $i \sim 80^{\circ e}$ \\
2MASSJ 1628137-243139 & $16: 28: 13.7,-24: 31: 39.00$ & II & $i \sim 86^{\circ}$ \\
HV Tau & $04: 38: 35.280,+26: 10: 39.88$ & II & multiple system $\left(i \sim 84^{\circ g}\right)$ \\
HK Tau & $04: 31: 50.900,+24: 24: 17.00$ & II & binary $\left(i \sim 85^{\circ h}\right)$ \\
UY Aur & $04: 51: 47.31,+30: 47: 13.9$ & II & $i \sim 42^{\circ}$ \\
\hline
\end{tabular}

Notes. ${ }^{(a)}$ Coordinate of the center of the $1^{\prime}$ aperture. ${ }^{(b)}$ Inclination of the disk estimated by Tobin et al. (2008). ${ }^{(c)}$ Judged from the similarity of its N3 image and spectrum to those of L1527 and IRAS 04302. ${ }^{(d)}$ Wolf et al. (2003). ${ }^{(e)}$ Hodapp et al. (2004). ${ }^{(f)}$ Grosso et al. (2003). ${ }^{(g)}$ Inclination of HV Tau C (Monin \& Bouvier 2000). ${ }^{(h)}$ Inclination of HK Tau B (Stapelfeldt et al. 1998).

Both IRAS 04302 and L1527 (IRAS 04368+2557) are edgeon YSOs in a transient state from class 0 to class I (André et al. 2000). The YSO L1527 is of special interest from a chemical point of view; it is one of two YSOs where high abundances of carbon-chain species are observed. Carbon chains are considered to be formed by warm carbon-chain chemistry (WCCC): gasphase reactions of sublimated $\mathrm{CH}_{4}$ (Sakai et al. 2008; Aikawa et al. 2008). The large organic species detected towards several low-mass YSOs (e.g. IRAS 16293) are not, however, detected towards L1527 (Sakai et al. 2008). Since the large organic species are formed from $\mathrm{CH}_{3} \mathrm{OH}$, while carbon chains are formed from $\mathrm{CH}_{4}$, it is important to investigate ice bands, including the $\mathrm{C}-\mathrm{H}$ bands, towards L1527, to determine whether its ice composition significantly differs from those of other low-mass YSOs.

The YSO IRC-L1041-2 was initially in our target list of background stars (i.e. field stars behind molecular clouds) (Noble et al., in prep.). The N3 image shows a faint object with nebulosity, which is similar to L1527. The SST observations also detected a YSO with nebulosity (T. L. Bourke, priv. comm.). The presence of a dark dust lane indicates that the YSO has a high inclination, close to edge-on.

The YSOs 2MASS J1628137 and ASR41 are edge-on class II objects. 2MASS J1628137 is accompanied by a tenuous $\left(4 \times 10^{-4} M_{\odot}\right)$ envelope (Grosso et al. 2003). Hodapp et al. (2004) performed detailed modeling of the scattering and radiative transfer of ASR 41 and concluded that the $K$-band image can be well reproduced by a protoplanetary disk embedded in a lowdensity cloud.

HK Tau B and HV Tau C are edge-on class II objects, in the HK Tau binary and HV Tau triplet systems, respectively. We initially aimed to obtain spectra of these edge-on objects, but the spectra were found to be contaminated and dominated by the bright primaries, because the binary separations are small (see Sects. 4.1 and 5). The ice absorption features towards HK Tau B and HV Tau $\mathrm{C}$ might still be observable, although the derivation of ice column densities is not straightforward. For a comparison with disks that are not edge-on, we also observed UY Aur, which is a binary system with a separation of $0.9^{\prime \prime}$ accompanied by a circumbinary disk. The inclination of the disk is $\sim 42^{\circ}$ (Hioki et al. 2007).

\section{Data reduction and analysis}

\subsection{Reduction of the spectral image}

The reduction pipeline provided by the AKARI project team, IRC_SPECRED (Ohyama et al. 2007), is unsuitable for our targets, because it automatically defines the area surrounding selected sources as "sky", an area that is often contaminated by nebulosity in our observations.

To derive spectra from the data, we developed our own procedure, referring to the reduction method by Sakon et al. (2007). Firstly, we made a cosmic-ray-free dark for each target by taking the median of three dark frames; two dark frames were taken during the observation of each target, and one additional dark frame was taken from the preceding or subsequent observation in the AKARI archive. This procedure was necessary, because some frames are heavily damaged by cosmic rays. The dark was subtracted from the N3 image and spectral images.

The eight spectral images were slightly shifted in relation to each other by the attitude drift of the satellite. The task IRC_SPECRED measures the shift of each image relative to the fourth image. Referring to this measurement, we selected images with a relative shift of within 0.5 pixels, in both spatial and spectral directions. Images with larger shifts were discarded. Cosmic rays were removed by subtracting the median of the possible combinations of three frames from the selected frames. We then stack and average these cosmic-ray-free spectral images.

The local-sky region was selected by carefully avoiding nebulosity, field stars, and bad pixels. In the case of L1527, there is no "sky" region, because the nebulosity extends all over the $1^{\prime} \times 1^{\prime}$ aperture (Fig. 1). Since we performed slitless spectroscopy, the spectral image of the nebulosity is a convolution of the nebula spectrum with the brightness distribution, and should not be considered as "sky". We selected from the AKARI archive a $1^{\prime} \times 1^{\prime}$ spectral image that can be used as sky; i.e. a dark region observed at a similar period and coordinate to those of L1527.

After the sky subtraction, we defined the spectrum region of each star in the spectral image, referring to the coordinates of the objects and the N3 image. The signal was integrated over eight pixels (12 arcsec) in the spatial direction. As an alternative approach, Noble et al. (in prep.) fit the signal at each wavelength with the PSF and integrated the signal. We confirmed that the result of the eight-pixel integration agrees well with this more sophisticated approach. We note that the spectrum is not perfectly aligned with the rows of the pixels. We adopted the formulation as the tilt of the spectrum

$Y=-0.00746929\left(X-X_{0}\right)+Y_{0}$,

where $X$ and $Y$ are the pixel number in the dispersion direction and spatial direction respectively, and $Y_{0}$ is the center of the spectrum at an arbitrary position, $X_{0}$, in the dispersion direction.

In Fig. 1, nebulosity is clearly seen towards L1527, IRCL1041-2, IRAS 04302, and ASR 41. For these objects, we set $Y_{0}$ in Eq. (1) as the brightest position in the spatial direction at a wavelength $\left(X_{0}\right)$ in the spectrum image, and integrated the 
spectrum over eight pixels in the spatial direction at each wavelength (Fig. 1). One exception is L1527; since the central pointlike source is apparent and fainter than the nebulosity, we set $Y_{0}$ as the central (faintest) position in the spatial direction, and integrated the signal over four pixels in the spatial direction (Fig. 1).

The integrated signal was divided by the aperture correction factor and response function before being converted into a spectrum. The response function was determined by the observation of a standard star (KF09T1). Wavelength calibration was done by the AKARI project team using the slit spectroscopy observation of standard stars. In our observations, we determined the wavelength of the spectrum by measuring the shift between the slit and the direct light position of the target in the N3 image.

According to the IRC team, the pixel-wavelength relation is a linear function. This linear correlation is confirmed in the wavelength range $2.5-4.5 \mu \mathrm{m}$, although the calibration is less certain at longer wavelengths. The $\mathrm{CO}$ absorption peak $(\sim 4.67 \mu \mathrm{m})$ is shifted to shorter wavelengths by 1-2 pixels in our data. Since similar shifts in this wavelength are reported in other IRC data, we shifted the data at $\geq 4.5 \mu \mathrm{m}$ by $1-2$ pixels towards longer wavelengths.

The error in flux was evaluated as follows. Firstly, we estimated the noise level in the "sky" region and converted it into an error in flux using the response function. Secondly, we estimated the flux errors caused by the wavelength assignment; we calculated fluxes by assuming that the shift between the slit and the direct light position of the target is 0.5 pixels larger/smaller. We then adopted the maximum of these errors.

\subsection{Absorption features}

To evaluate the ice absorption profile quantitatively, we normalized the spectrum with the continuum, which is assumed to be a second order polynomial, and was determined by referring to the three wavelength regions $\sim 2.6 \mu \mathrm{m}, 3.4-3.8 \mu \mathrm{m}$, and $4.2-4.9 \mu \mathrm{m}$. The choice of wavelengths differed slightly among objects to ensure a good fit to all spectra.

Template absorption profiles were obtained from "data base 2007" at Raymond \& Beverly Sackler Laboratory for Astrophysics ${ }^{1}$. The database summarizes the absorption profiles of ice species measured in laboratories. Gerakines et al. (1995, 1996) measured the profiles and determined band strengths of various astrophysical ice analogs. From the database of Gerakines et al. (1995, 1996), we adopted profiles of pure ices at $10 \mathrm{~K}$ unless otherwise stated. In reality, interstellar ice is a mixture of various components such as $\mathrm{H}_{2} \mathrm{O}, \mathrm{CO}$, and $\mathrm{CO}_{2}$, and the peak position and shape of the absorption features change with the abundance ratio of the components. However, our spectral resolution is insufficiently high to perform such a detailed analysis of the ice mixture.

The profiles of the $4.27 \mu \mathrm{m} \mathrm{CO}_{2}$ band and the $4.67 \mu \mathrm{m}$ $\mathrm{CO}$ band depend strongly on grain shape (Ehrenfreund et al. 1997). We adopted the continuously distributed ellipsoids (CDE) grain model for the CO band feature (Ehrenfreund et al. 1997; Boogert et al. 2002; Pontoppidan et al. 2003a). For the $\mathrm{CO}_{2}$ band, on the other hand, we adopted the spectrum of Gerakines et al. (1995), which is uncorrected for the grain shape, since it gives a good fit to our observational data. The pure $\mathrm{CO}_{2}$ feature with grain-shape (CDE) corrections agrees with neither our data nor the $\mathrm{CO}_{2}$ ice feature observed by ISO towards highmass YSOs, which indicates that $\mathrm{CO}_{2}$ ice is not pure in interstellar medium. The ISO observations of the $4.27 \mu \mathrm{m}$ feature

\footnotetext{
1 http://www.strw.leidenuniv.nl/ lab/
}

indicated that the $\mathrm{CO}_{2}$ ice is primarily in a polar $\left(\mathrm{H}_{2} \mathrm{O}\right.$-rich) component of the ices (Whittet et al. 1998). However, to interpret our observation, we also need to introduce an apolar component to reproduce the blue wing at $\sim 4.2 \mu \mathrm{m}$. Since our spectral resolving power is low, we simply use the spectrum of Gerakines et al. (1995) as a template, rather than combining multiple components of ice mixture with grain shape corrections. We also adopted the $3.05 \mu \mathrm{m} \mathrm{H}_{2} \mathrm{O}$ band of Gerakines et al. (1995), since it is little affected by the grain shape.

The spectral resolution of IRC, $\sim 100$, is not high enough to fully resolve narrow bands such as $\mathrm{CO}$ and $\mathrm{CO}_{2}$. We fitted these narrow bands using the following method: we considered the absorbance of ice from the database and convert it into a normalized flux spectrum, assuming the optical depth of ice along the line of sight. The spectrum was then convolved with the instrumental line profile and re-binned to the resolving power of AKARI, to be compared with the observed spectrum. We vary the optical depth of ice until the best-fit is found by least squares fitting. Broad absorption features such as the $\mathrm{H}_{2} \mathrm{O}$ band at $3.05 \mu \mathrm{m}$ were not significantly altered by the convolution.

In N3 images $(2.7-3.8 \mu \mathrm{m})$, class 0-I objects (L1527, IRCL1041-2, and IRAS 04302) are more extended than a point source. Among the three objects, L1527 is the most extended, and IRAS 04302 is the least extended. Since the IRC spectrograph is slitless, the spectral resolving power depends sensitively on the spatial extent of the source. Assuming that the projected column density of ice in front of the extended sources is constant, the observed band profile is a convolution of the ice absorptions with the spatial distribution of the light source in the dispersion direction. For L1527, we integrated the central four pixels of the N3 image in the spatial direction around the source position to derive the spatial distribution of the light source in the dispersion direction (N3 profile, hereafter), with which the laboratory ice spectrum was convolved. This convolution better reproduces the observed features of $\mathrm{CO}$ and $\mathrm{CO}_{2}$ than the convolution with the instrumental line profile. For IRC-L1041-2 and IRAS 04302, however, we convolved the ice features with the instrumental line profile, which reproduces the observed spectrum better than the N3 profile. Since the instrumental line profile at $4 \mu \mathrm{m}$ (N4 band) is narrower than that of the N3 band, the $\mathrm{N} 3$ profile might be too broad to be applied to $\mathrm{CO}$ and $\mathrm{CO}_{2}$ features. Unfortunately, imaging observation was not performed in the N4 band.

Once we determined the best-fit absorption feature, we used the original, pre-convolution profile to calculate the the column density $N$ of ice

$N=\int \frac{\tau \mathrm{d} v}{A}$

where $A$ is the band strength (e.g. Whittet et al. 1993). We adopted the band strength listed in Table 2 of Gibb et al. (2004), unless otherwise stated (Table 2). The CO ice column density of the CDE grain model was calculated to be

$N=6.03 \tau_{\max } A^{-1}$,

where $A=1.1 \times 10^{-17} \mathrm{~cm} \mathrm{molec}^{-1}$ and $\tau_{\max }$ is the optical depth at the wavelength of the absorption peak (Pontoppidan et al. 2003a).

Since the spectral resolution is low, and since some lines are saturated, it was difficult to estimate the errors in the ice column densities accurately. In the present paper, the column density error was evaluated by simply calculating the error in the absorption area. For $\mathrm{CO}_{2}$, we calculated the absorption area $\int \tau \mathrm{d} v$ at 
$4.2-4.3 \mu \mathrm{m}$ and its error caused by the flux error. Assuming that the absorption area had an error of $11 \%$, and that the $\mathrm{CO}_{2}$ column density was estimated to be $10.0 \times 10^{17} \mathrm{~cm}^{-2}$, for example, the column density error was calculated as $1.1 \times 10^{17} \mathrm{~cm}^{-2}$. For $\mathrm{H}_{2} \mathrm{O}$, we estimated the error by calculating the absorption area between $2.8-3.2 \mu \mathrm{m}$. If the red wing was much broader than the laboratory spectrum, we estimated the error from the absorption area at $2.8-3.0 \mu \mathrm{m}$. For L1527, towards which the $\mathrm{H}_{2} \mathrm{O}$ band is saturated, we used the wavelength range $\sim 2.8-2.9 \mu \mathrm{m}$. The absorption at $\sim 4.7 \mu \mathrm{m}$ is a combination of the absorptions of $\mathrm{CO}, \mathrm{XCN}$, and $\mathrm{CO}$ gas (Sect. 4.5). Here, the error is evaluated by calculating the absorption area at $4.65-4.7 \mu \mathrm{m}$ for $\mathrm{CO}$ and $4.57-4.65 \mu \mathrm{m}$ for XCN. Uncertainties in ice column densities originating from the choice of the convolution function (instrumental line profile or N3 profile) are discussed in Sect. 4.

The derivation of ice column densities described above implicitly assumes a simple geometry: a point light source behind a uniform absorbing material. Edge-on YSOs have more complicated structures. Stellar light scattered by the outflow cavity can be an extended light source (e.g. Tobin et al. 2008), even if it looks like a point source with a PSF of a few arc seconds, and the column density of the absorbing material (envelope and/or disk) also varies spatially. More accurate estimates of the ice column densities requires the radiative transfer calculation of twodimensional (2D) or three-dimensional (3D) models of envelope and disk structure (Pontoppidan et al. 2005). We compare the observed spectra with existing model calculations and discuss the location of ice in Sect. 5.

\section{Results}

\subsection{Spectrum}

Figure 2 shows the derived spectra of our target YSOs. The spectrum of L1041-2 resembles that of L1527, which suggests a similarity in the structure and evolutionary stage of these objects. The spectrum of IRAS 04302, on the other hand, is less reddened than L1527. It might be in a more evolved stage than L1527 and L1041-2.

2MASS J1628137-243139 is very faint and is almost at the faintest limit for which we can derive a spectrum. The spectrum of ASR 41 shows a sharp decline at $\sim 4.5 \mu \mathrm{m}$ and is noisy at longer wavelengths because of the column pulldown caused by a bright star in the $10^{\prime} \times 10^{\prime}$ aperture.

HV Tau is a triplet system: HV Tau A and HV Tau B are a binary system with a separation of 0.0728" (Simon et al. 1998), and our target edge-on disk, HV Tau C, is separated by $\sim 4^{\prime \prime}$ from the binary (Woitas \& Leinert 1998). Since the FWHM of the AKARI PSF is $\sim 4.3^{\prime \prime}$ in the NIR and the PSF is not circular, it is difficult to disentangle the HV Tau C spectrum from that of the system. Hence, we integrated the spectral image over an eightpixel width. The spectrum is dominated by HV Tau A and B at 2.5-4.5 $\mu \mathrm{m}$, as they are brighter than HV Tau C by $4.22 \mathrm{mag}$ in the $K$ band and $>1.71 \mathrm{mag}$ in the $L$ band. At $4.5 \mu \mathrm{m}$, on the other hand, the contribution of HV Tau C may not be negligible, because HV Tau $\mathrm{C}$ is as bright as the HV Tau AB system in the $N$ band (Woitas \& Leinert 1998). HK Tau is also a binary system. The edge-on object, HK Tau B, is a faint binary. Unfortunately, it is impossible to disentangle the spectrum of HK Tau B from the primary, because the two stars almost align in the dispersion direction. It is disappointing that we were unable to discern the spectra of HV Tau C and HK Tau B. The absorption features are, however, still apparent in our spectra. We compare these
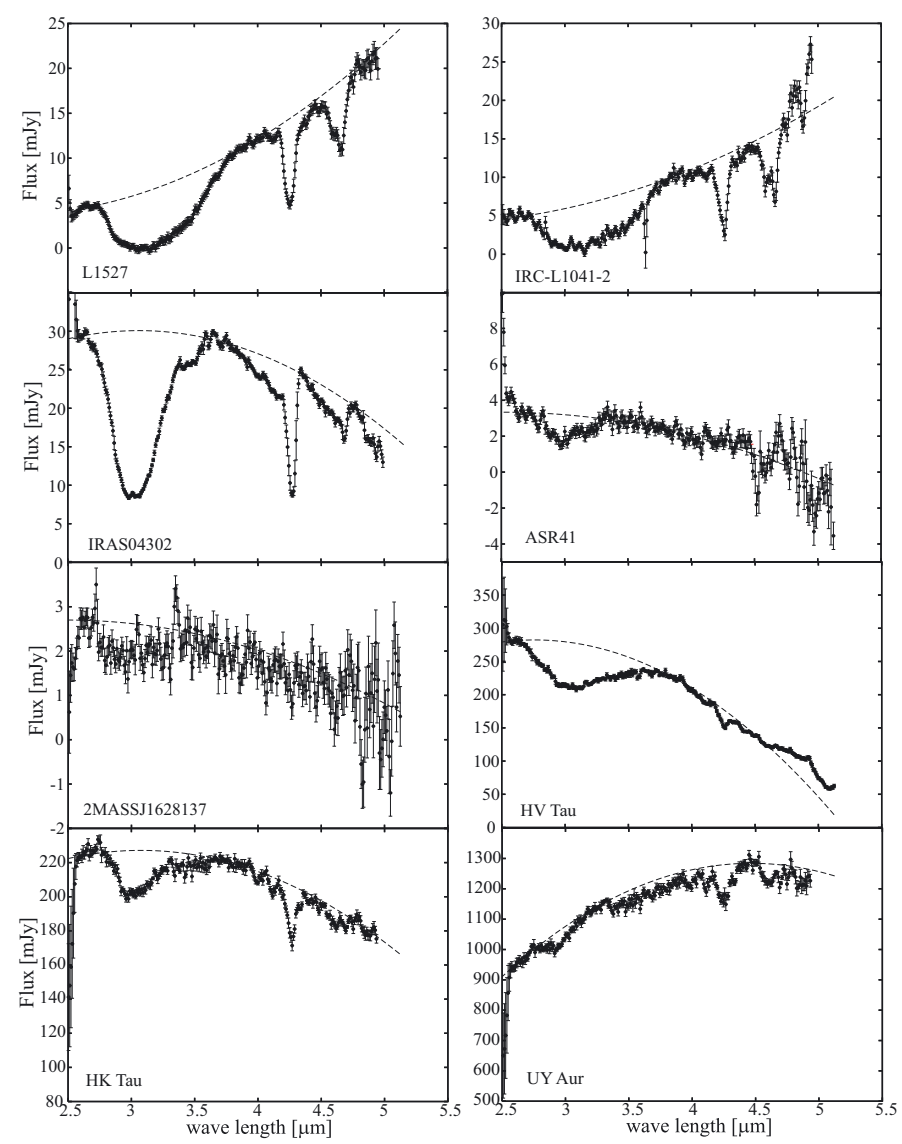

Fig. 2. The derived spectra of target YSOs in the NIR (closed circles with error bars). The continuum (dashed line) is determined by the second-order polynomial fitting of the spectrum.

absorptions with Subaru observations towards the HV Tau C and HK Tau B by Terada et al. (2007) in the following discussion.

The dashed lines in Fig. 2 depict the continuum. While the continuum varies between objects, reflecting their structure and evolutionary stage, we can clearly see absorption bands of $\mathrm{H}_{2} \mathrm{O}(3.05 \mu \mathrm{m}), \mathrm{CO}_{2}(4.27 \mu \mathrm{m})$, and $\mathrm{CO}(4.67 \mu \mathrm{m})$ in most of the spectra. The assignment and fitting of each ice band are performed in the following subsections.

\section{2. $\mathrm{H}_{2} \mathrm{O}$ ice}

Figure 3 shows the spectra normalized by the continuum. The vertical axis is the optical depth of the absorption. Towards L1527 and IRC-L1041-2, the $3.05 \mu \mathrm{m}$ water band is clearly saturated; the flux at the absorption peak is almost zero (Fig. 2). We fitted the spectra in the range $2.9-3.1 \mu \mathrm{m}$ with the laboratory spectrum of pure $\mathrm{H}_{2} \mathrm{O}$ ice at $10 \mathrm{~K}$ (Gerakines et al. 1995) (red lines in Fig. 3). We also fitted the observed feature with the pure $\mathrm{H}_{2} \mathrm{O}$ ice feature at $160 \mathrm{~K}$. The agreement is much worse than for the ice at $10 \mathrm{~K}$, which suggests that the bulk of the observed $\mathrm{H}_{2} \mathrm{O}$ ice is not annealed.

Interestingly, the profiles fitted at $2.9-3.1 \mu \mathrm{m}$ (solid lines in Fig. 3) reproduce the shallow hollow at $4.5 \mu \mathrm{m}$, which is the $\mathrm{O}-\mathrm{H}$ combination mode (Boogert et al. 2000). While the $3.05 \mu \mathrm{m}$ band is saturated, the $4.5 \mu \mathrm{m}$ band suggests that the $\mathrm{H}_{2} \mathrm{O}$ column density cannot be significantly larger than we estimated.

Table 2 summarizes the column density of $\mathrm{H}_{2} \mathrm{O}$ ice derived by fitting the $3.05 \mu \mathrm{m}$ band. The class 0-I objects L1527, IRAS 04302, and IRC-L1041-2 clearly have much higher water 

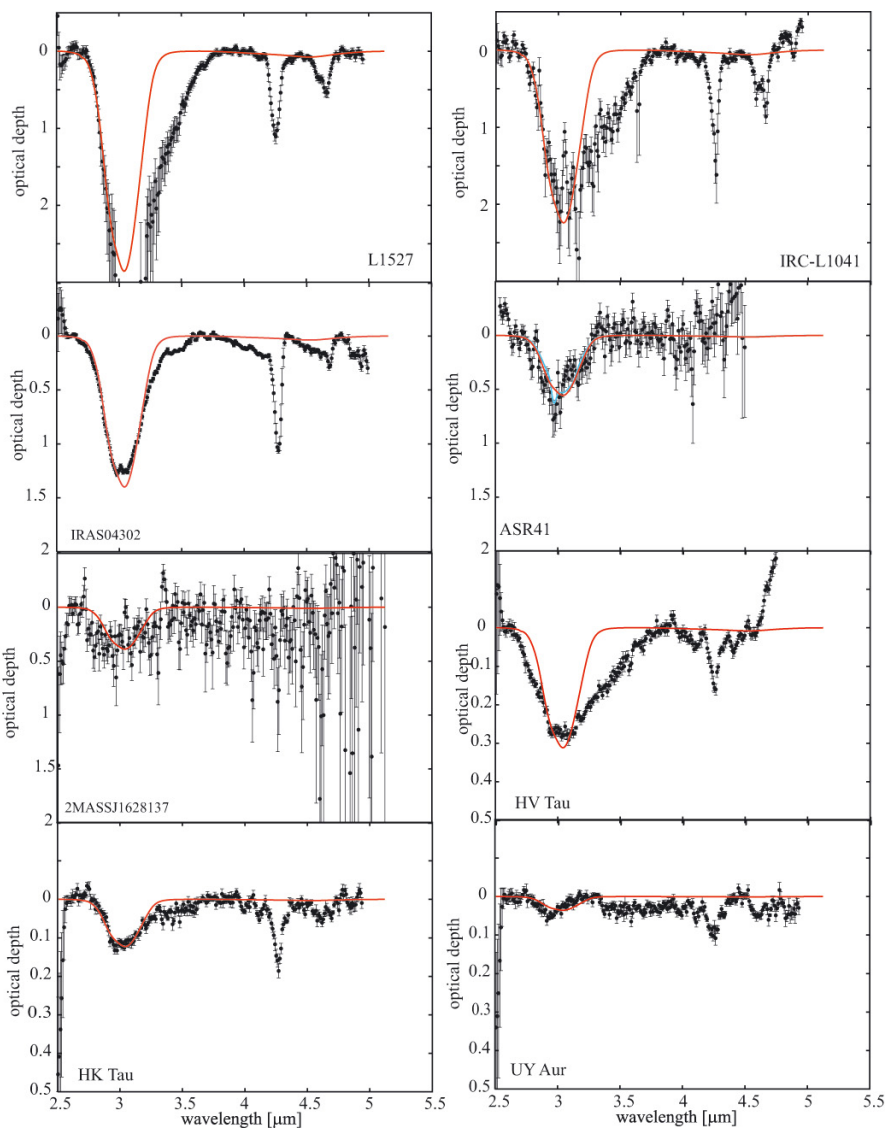

Fig. 3. Closed circles with error bars depict the YSO spectrum normalized by the continuum. The absorption feature at $3.05 \mu \mathrm{m}$ is fitted by the laboratory spectrum of pure $\mathrm{H}_{2} \mathrm{O}$ ice at $10 \mathrm{~K}$ (red line) (see text).

column densities than the other targets, probably because of the larger mass of their circumstellar material and geometrical effects (Sect. 5).

The observed profiles show a red wing $(>3.1 \mu \mathrm{m})$, which is often observed in molecular clouds. The shape and depth of the red wing relative to the peak $(3.05 \mu \mathrm{m})$ vary significantly among objects; it is especially deep towards L1527 and L1041-2. The wing is generally attributed to the scattering by large grains with ice mantles (e.g. Léger et al. 1983). The size of the grains responsible for the red wing is $\lambda / 2 \pi \sim 0.5 \mu \mathrm{m}$.

ASR 41 has a double-peaked profile at the absorption peak $\sim 3 \mu \mathrm{m}$. We reduced each frame of the spectral image to confirm that the double-peaked profile is not caused by noise in a specific frame. The double-peaked profile remained, even if we changed the combination of frames in the stacking. We fitted the profile with (i) the $\mathrm{H}_{2} \mathrm{O}$ band alone (red line in Fig. 3); and (ii) the $\mathrm{H}_{2} \mathrm{O}$ band plus an $\mathrm{NH}_{3}$ band $(2.98 \mu \mathrm{m})$ (blue line in Fig. 3). Although the blue line seems to fit the observed profile slightly better, it results in an unreasonably high column density of $\mathrm{NH}_{3}\left(2 \times 10^{18} \mathrm{~cm}^{-2}\right)$ compared with the $\mathrm{H}_{2} \mathrm{O}$ column density $\left(7.8 \times 10^{17} \mathrm{~cm}^{-2}\right)$, and the bump at $3.1 \mu \mathrm{m}$ is not fitted well. While the double-peaked profile seems to be robust, it could still be noise, since the flux at the absorption peak is only 1-2 mJy. We therefore prefer to fit the $3 \mu \mathrm{m}$ absorption profile with the $\mathrm{H}_{2} \mathrm{O}$ band alone.

In the polynomial fitting of the HV Tau continuum, we ignored the spectrum at $>4.5 \mu \mathrm{m}$, since the gradual rise of the spectrum at longer wavelengths may be caused by HV Tau C, while shorter wavelengths are dominated by HV Tau AB. The absorption band at $3 \mu \mathrm{m}$ is much broader than the laboratory profile. While the red wing may be caused by scattering, the blue wing may be caused by the combination of the spectra of HV Tau A and B. The binary is close enough ( $\left.\sim 0.07^{\prime \prime}\right)$ to be considered as a point source in our observation, but the objects have similar brightnesses and different colors (Simon et al. 1998). We tentatively fitted the absorption peak with the laboratory data to derive the water ice column density.

Terada et al. (2007) detected deep $\mathrm{H}_{2} \mathrm{O}$ absorption towards the edge-on disks of HV Tau C and HK Tau; they concluded that these absorptions originate in the disk ice. If there were no foreground clouds with ice, our spectra should be a combination of the edge-on disk with ice features and bright primaries without absorption. The observed $3 \mu \mathrm{m}$ absorption of HV Tau is, however, too deep to be accounted for by the disk ice alone; while the flux of $\mathrm{HV}$ tau $\mathrm{C}$ is $7.75 \mathrm{mJy}$ at $K$ band and $7.14 \mathrm{mJy}$ at $L^{\prime}$ band, the $3 \mu \mathrm{m}$ absorption is as deep as $>50 \mathrm{mJy}$. There must be a contribution of ice absorption in foreground clouds. The $\mathrm{H}_{2} \mathrm{O}$ column density in Table 2 should be considered as upper limits to the ice in foreground clouds, since we do not distinguish between the disk ice and foreground ice. However, the $3 \mu \mathrm{m}$ absorption of HK Tau might be due to the disk ice of HK Tau B; the flux of HK Tau B is $15.9 \mathrm{mJy}$ at $2.2 \mu \mathrm{m}$ and $9.85 \mathrm{mJy}$ at $3.8 \mu \mathrm{m}$, while the absorption is $\$ 20 \mathrm{mJy}$. If we were to assume that the flux of the primary is $200 \mathrm{mJy}$, subtract it from the spectrum, and fit the $3 \mu \mathrm{m}$ absorption by the $\mathrm{H}_{2} \mathrm{O}$ ice feature, the ice column density would be $3 \times 10^{18} \mathrm{~cm}^{-2}$, which is consistent with the value derived by Terada et al. (2007) $\left(3.31 \times 10^{18} \mathrm{~cm}^{-2}\right)$.

\section{3. $\mathrm{CH}$ band at $3.5 \mu \mathrm{m}$}

While the long wavelength wing of $\mathrm{H}_{2} \mathrm{O}$ absorption is generally attributed to scattering by large grains with ice mantles, it might also represent the stretching mode of $\mathrm{C}-\mathrm{H}$ bond. Among various possible carriers of the $\mathrm{C}-\mathrm{H}$ bond, $\mathrm{CH}_{3} \mathrm{OH}$ ice has long been observed via the substructures at $3.47 \mu \mathrm{m}$ and $3.53 \mu \mathrm{m}$ (e.g. Grim et al. 1991; Brooke et al. 1996). While the earlier works are mostly restricted to high-mass and intermediate YSOs, Pontoppidan et al. (2003b) identified the $3.5 \mu \mathrm{m}$ feature towards three low-mass YSOs (out of 40 sources) as $\mathrm{CH}_{3} \mathrm{OH}$ ice. Boogert et al. (2008) detected the C-O stretching mode of $\mathrm{CH}_{3} \mathrm{OH}$ at $9.7 \mu \mathrm{m}$ towards 12 low-mass YSOs (out of 41 sources) using Spitzer. Boogert et al. (2011) detected the $3.53 \mu \mathrm{m}$ band of $\mathrm{CH}_{3} \mathrm{OH}$ towards several field stars behind isolated dense cores; the $\mathrm{CH}_{3} \mathrm{OH} / \mathrm{H}_{2} \mathrm{O}$ ratio ranges from $5 \%$ to $20 \%$. The ratio of $\mathrm{CH}_{3} \mathrm{OH}$ to $\mathrm{H}_{2} \mathrm{O}$ column densities varies considerably among objects.

Another possible carrier of the $\mathrm{C}-\mathrm{H}$ bond is $\mathrm{CH}_{4}$, whose absorption bands at $3.32 \mu \mathrm{m}$ and $7.67 \mu \mathrm{m}$ have been detected towards high-mass YSOs (e.g. Lacy et al. 1991; Boogert et al. 2004). Öberg et al. (2008) detected a $7.7 \mu \mathrm{m}$ absorption feature, which is attributed to $\mathrm{CH}_{4}$, towards 25 sources out of 52 lowmass YSOs. Since $\mathrm{CH}_{3} \mathrm{OH}$ and $\mathrm{CH}_{4}$ are considered to be the precursors of large organic molecules in hot corinos and WCCC, respectively, it is important to constrain their abundances using the spectrum around $3.5 \mu \mathrm{m}$.

Figure 4 shows the spectra of L1527, IRAS 04302, and HK Tau around $3.5 \mu \mathrm{m}$. While the spectrum of L1527 is smooth, those of IRAS 04302 and HK Tau contain absorption features. These features, however, do not match either $\mathrm{CH}_{3} \mathrm{OH}$ or $\mathrm{CH}_{4}$. A potential complication to the identification of, in particular, the $\mathrm{CH}_{4} 3.32 \mu \mathrm{m}$ band and to a lesser degree the $\mathrm{CH}_{3} \mathrm{OH} 3.53 \mu \mathrm{m}$ band, is the possible presence of the $3.3 \mu \mathrm{m}$ PAH emission feature and the $\operatorname{HI} \operatorname{Pf} \delta$ line at $3.297 \mu \mathrm{m}$. We can indeed see an 
Table 2. Absorption peak, band strength, and column densities of ices towards objects.

\begin{tabular}{lccccc}
\hline \hline & $\mathrm{H}_{2} \mathrm{O}$ & $\mathrm{HDO}$ & ${ }^{12} \mathrm{CO}_{2}$ & $\mathrm{CO}$ & $\mathrm{XCN}$ \\
\hline Absorption peak $[\mu \mathrm{m}]$ & 3.05 & 4.07 & 4.27 & 4.67 & 4.62 \\
${\text { Band strength } 10^{-17} \mathrm{~cm} \text { molecule }}^{-1}$ & 20 & 4.3 & 7.6 & see text $^{17}$ & 5.0 \\
\hline Column density & $10^{17}\left[\mathrm{~cm}^{-2}\right]$ & $10^{17}\left[\mathrm{~cm}^{-2}\right]$ & $10^{17}\left[\mathrm{~cm}^{-2}\right]$ & $10^{17}\left[\mathrm{~cm}^{-2}\right]$ & $10^{17}\left[\mathrm{~cm}^{-2}\right]$ \\
\hline L1527(04368+2557) & $47 \pm 13$ & $0.96(2.0 \%)^{a}$ & $10 \pm 1.1(21 \%)$ & $18 \pm 2.6(38 \%)$ & $1.5 \pm 0.22(3.2 \%)$ \\
IRC-L1041-2 & $39 \pm 6.6$ & $3.7(9.5 \%)$ & $9.4 \pm 1.6(24 \%)$ & $18 \pm 4.0(46 \%)$ & $2.5 \pm 0.48(6.4 \%)$ \\
IRAS 04302+2247 & $24 \pm 0.82$ & $5.3(2 \%)$ & $6.8 \pm 0.64(28 \%)$ & $3.1 \pm 0.55(13 \%)$ & $0.53 \pm 0.064(2.2 \%)$ \\
ASR 41 & $7.8 \pm 2.2$ & - & - & - & - \\
2MASSJ 1628137-243139 & $6.7 \pm 2.6$ & - & - & - & - \\
HV Tau & $5.4 \pm 0.23$ & $1.0(19 \%)$ & $0.72 \pm 0.11(13 \%)$ & - & - \\
HK Tau & $2.1 \pm 0.23^{b}$ & - & $0.9 \pm 0.11(43 \%)$ & - & - \\
UY Aur & $0.61 \pm 0.20$ & - & $0.54 \pm 0.086(89 \%)$ & & - \\
\hline
\end{tabular}

Notes. ${ }^{(a)}$ Abundance relative to $\mathrm{H}_{2} \mathrm{O}$ ice. ${ }^{(b)}$ If the absorption originates in $\mathrm{HK}$ Tau B, ice column density is $3 \times 10^{18} \mathrm{~cm}^{-2}$ (see text).

emission feature at $3.3 \mu \mathrm{m}$ in HK Tau that could be ascribed to the PAH band. In the following, we derive upper limits to the column densities of $\mathrm{CH}_{3} \mathrm{OH}$ and $\mathrm{CH}_{4}$, keeping in mind that the low spectral resolving power of our spectra may blend adjacent emission and absorption bands.

The thick solid lines in Fig. 4 depict the upper limits on the $\mathrm{CH}_{4}$ absorption depth. We convolved the pure $\mathrm{CH}_{4}$ ice feature at $10 \mathrm{~K}$ (Gerakines et al. 1995) with the instrumental line profile (IRAS 04302 and HK Tau) or the N3 profile (for L1527). The upper limits to the $\mathrm{CH}_{4}$ column density are $1.6 \times 10^{19} \mathrm{~cm}^{-2}$ (larger than the $\mathrm{H}_{2} \mathrm{O}$ column density), $3.1 \times 10^{17} \mathrm{~cm}^{-2}(13 \%$ relative to $\mathrm{H}_{2} \mathrm{O}$ ), and $1.6 \times 10^{17} \mathrm{~cm}^{-2}(76 \%)$ towards $\mathrm{L} 1527$, IRAS 04302, and HK Tau, respectively. Owing to the low flux, the upper limit towards L1527 is especially high.

The dashed lines show the upper limits to the $\mathrm{CH}_{3} \mathrm{OH}$ absorption depths, using a laboratory spectrum for pure $\mathrm{CH}_{3} \mathrm{OH}$ at $10 \mathrm{~K}$ (Gerakines et al. 1995), convolved with the instrumental line profile. While the $3.53 \mu \mathrm{m}$ feature is common among pure $\mathrm{CH}_{3} \mathrm{OH}$ ice and mixed ice $\left(\mathrm{H}_{2} \mathrm{O}+\mathrm{CH}_{3} \mathrm{OH}\right)$, the shape and depth of the $3.4 \mu \mathrm{m}$ feature depend on the composition of the mixture (e.g. Pontoppidan et al. 2003b). Hence, we derived upper limits to the $\mathrm{CH}_{3} \mathrm{OH}$ column density of the $3.53 \mu \mathrm{m}$ feature. The upper limits to the $\mathrm{CH}_{3} \mathrm{OH}$ column densities are $1.2 \times 10^{18} \mathrm{~cm}^{-2}(26 \%$ relative to $\left.\mathrm{H}_{2} \mathrm{O}\right), 1.0 \times 10^{18} \mathrm{~cm}^{-2}(42 \%)$, and $3.1 \times 10^{17} \mathrm{~cm}^{-2}$ (larger than the $\mathrm{H}_{2} \mathrm{O}$ column) towards L1527, IRAS 04302, and HK Tau, respectively.

\subsection{HDO ice}

In some objects, such as IRAS 04302, we can see absorption around $4.1 \mu \mathrm{m}$, where the OD stretching mode of HDO is observed in the laboratory (Dartois et al. 2003). Since the detection of HDO ice is very rare (Teixeira et al. 1999) and has not been confidently confirmed, we checked the response function carefully and confirmed that the $\sim 4.1 \mu \mathrm{m}$ feature is not caused by an artifact in the response function. Figure 5 shows the spectra of this wavelength region towards L1527, IRC-L1041-2, IRAS 04302, and HV Tau, which are fitted with a model of the amorphous HDO feature at $10 \mathrm{~K}$ : a Gaussian profile peaking at $4.07 \mu \mathrm{m}$ with a FWHM of $0.2 \mu \mathrm{m}$ (solid lines). The spectrum of L1527 is not fitted well with this Gaussian; the absorption has a peak at longer wavelength $(\sim 4.13 \mu \mathrm{m})$ and a narrower band width, which resembles an annealed, rather than an amorphous, HDO feature (Dartois et al. 2003). We fitted the L1527 spectrum with a Gaussian peaking at $4.13 \mu \mathrm{m}$ and FWHM of $0.1 \mu \mathrm{m}$ (dashed line in Fig. 5).
Although IRAS 04302 has the deepest and smoothest absorption around $4.1 \mu \mathrm{m}$, this result should be taken with caution. The spectrum also shows a broad absorption at $4.5-4.6 \mu \mathrm{m}$, which cannot be fitted well by the absorptions of $\mathrm{CO}$ and XCN (see Sect. 4.6). These two absorptions $(4.1 \mu \mathrm{m}$ and $4.5-4.6 \mu \mathrm{m})$ could be related; there could be an alternative explanation, rather than $\mathrm{HDO}, \mathrm{CO}$, and $\mathrm{XCN}$. The features themselves are, however, robust. We have two independent data sets of IRAS 04302, and the two broad absorptions appear in both data sets.

We integrated the fitted spectra to derive the column density of HDO (Table 2). The band strength was assumed to be $4.3 \times 10^{-17} \mathrm{~cm}$ molecule ${ }^{-1}$ (Dartois et al. 2003). Considering the small bumps and hollows that deviate from the HDO feature and the above discussion of IRAS 04302 spectrum, the HDO column densities should be interpreted with caution. However, at face value, the $\mathrm{HDO} / \mathrm{H}_{2} \mathrm{O}$ ratio ranges from $2 \%$ (L1527) to $22 \%$ (IRAS 04302). Except in the case of L1527, the ratios are much higher than those obtained in the previous observations and theoretical works: $\mathrm{HDO} / \mathrm{H}_{2} \mathrm{O} \leq 3 \%$ (Dartois et al. 2003; Parise et al. 2003, 2005; Aikawa et al. 2005).

\section{5. $\mathrm{CO}_{2}$ ice}

The $\mathrm{CO}_{2}$ band at $4.27 \mu \mathrm{m}$ is clearly detected towards our targets, except ASR41 and 2MASS J1628137. To subtract the $\mathrm{H}_{2} \mathrm{O}$ band at $4.5 \mu \mathrm{m}$, we divide the normalized spectrum by the best-fit $\mathrm{H}_{2} \mathrm{O}$ profile (red line in Fig. 3). The absorption at $4.27 \mu \mathrm{m}$ is then fitted by the laboratory $\mathrm{CO}_{2}$ profile convolved by the instrumental line profile (N3 profile for L1527) (solid line in Fig. 6). To illustrate how the profile is broadened by the instrumental line profile, the laboratory profile before convolution is shown in the panel of IRAS 04302 with the dashed line. While we adopted the pure $\mathrm{CO}_{2}$ profile measured by Gerakines et al. (1995) at $10 \mathrm{~K}$, the actual shape of the $\mathrm{CO}_{2}$ profile depends on various factors, such as its mixing with other ice species, grain shape, and annealing (Ehrenfreund et al. 1997; van Broekhuizen et al. 2006). We tried to fit the absorption bands with those of mixed ices of $\mathrm{CO}_{2}: \mathrm{CO}=1: 1$ at $15 \mathrm{~K}$, and $\mathrm{H}_{2} \mathrm{O}: \mathrm{CO}_{2}=100: 14$ at $10 \mathrm{~K}$, but the fits were worse than the pure $\mathrm{CO}_{2}$ ice of $10 \mathrm{~K}$. Although the observed profile might be better fitted by other mixtures and temperatures, we do not pursue this fitting, because the spectral resolution of the IRC is not high enough.

In regions close to the $\mathrm{YSO}, \mathrm{CO}_{2}$ sublimates at $\sim 70 \mathrm{~K}$. We note that $\mathrm{CO}_{2}$ gas has absorption lines in almost the same wavelength range as $\mathrm{CO}_{2}$ ice. Although the observed feature might be a combination of $\mathrm{CO}_{2}$ ice and gaseous $\mathrm{CO}_{2}$, the low spectral 

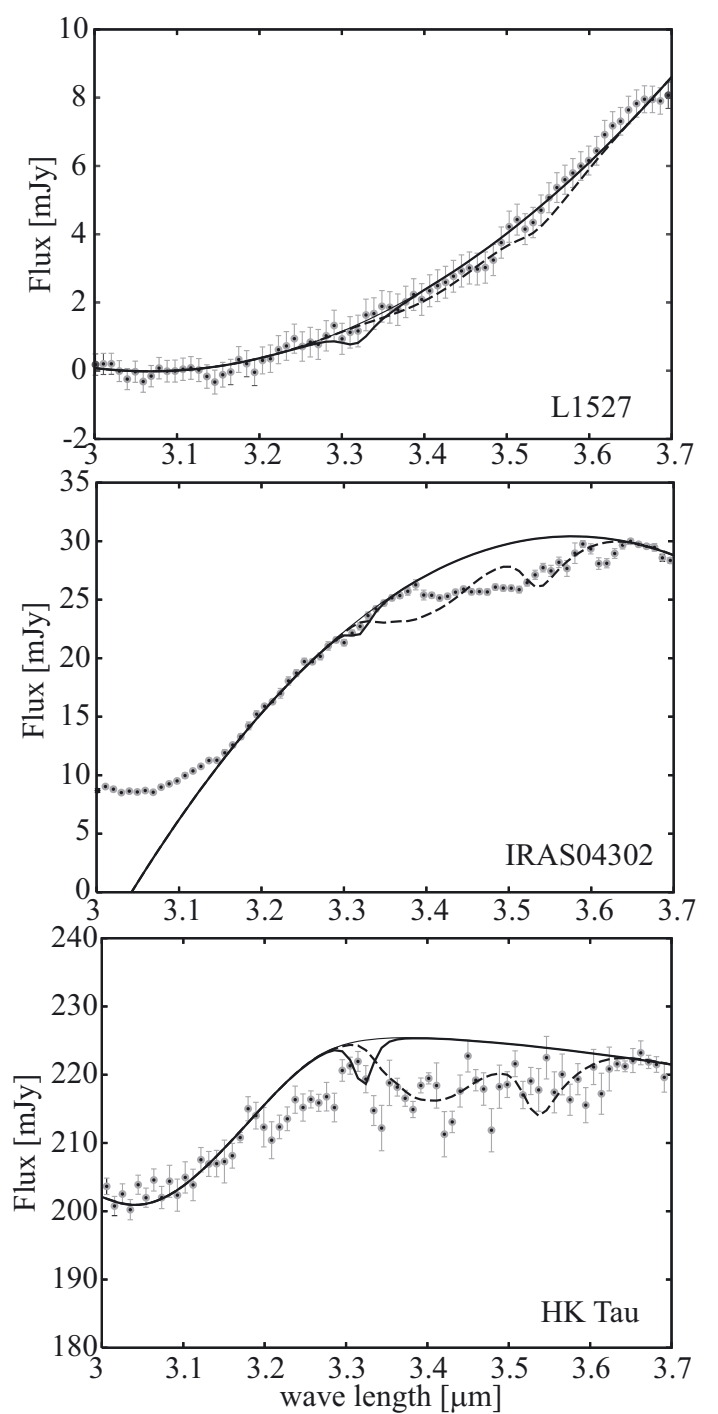

Fig. 4. Three-micron spectra of L1527, IRAS 04302, and HK Tau (gray dots with error bars). The thin solid lines in L1527 and IRAS 04302 are the local $(3.1-3.7 \mu \mathrm{m})$ continua fitted by second order polynomials, while the line in $\mathrm{HK}$ Tau is the best-fit absorption of $\mathrm{H}_{2} \mathrm{O}$ (Fig. 3). Solid and dashed lines depict the upper limits to the pure $\mathrm{CH}_{4}$ ice and pure $\mathrm{CH}_{3} \mathrm{OH}$ ice absorption depth, respectively.

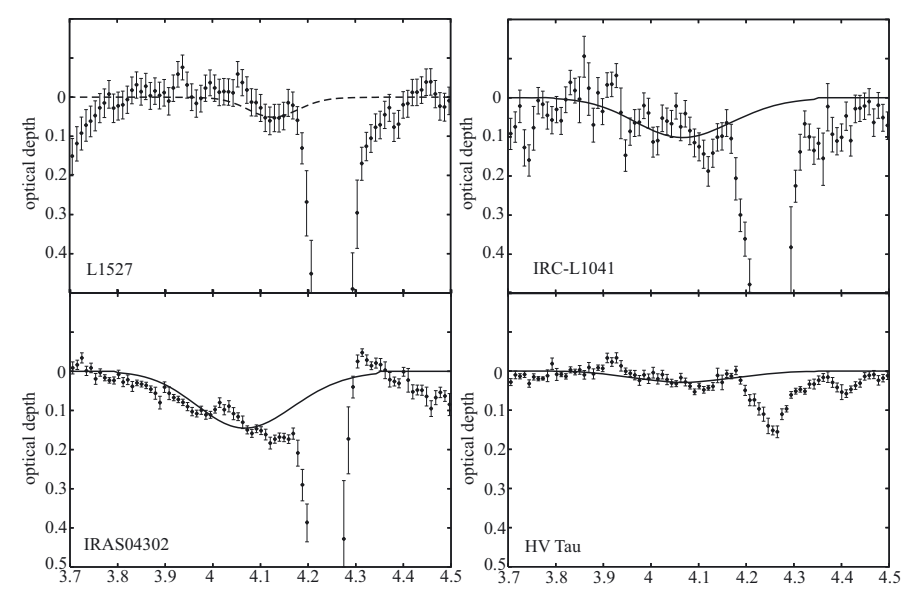

Fig. 5. Spectra around the HDO absorption band. The solid line is the Gaussian with FWHM of $0.2 \mu \mathrm{m}$ approximating the amorphous HDO ice feature, while the dashed line approximates the annealed HDO feature.

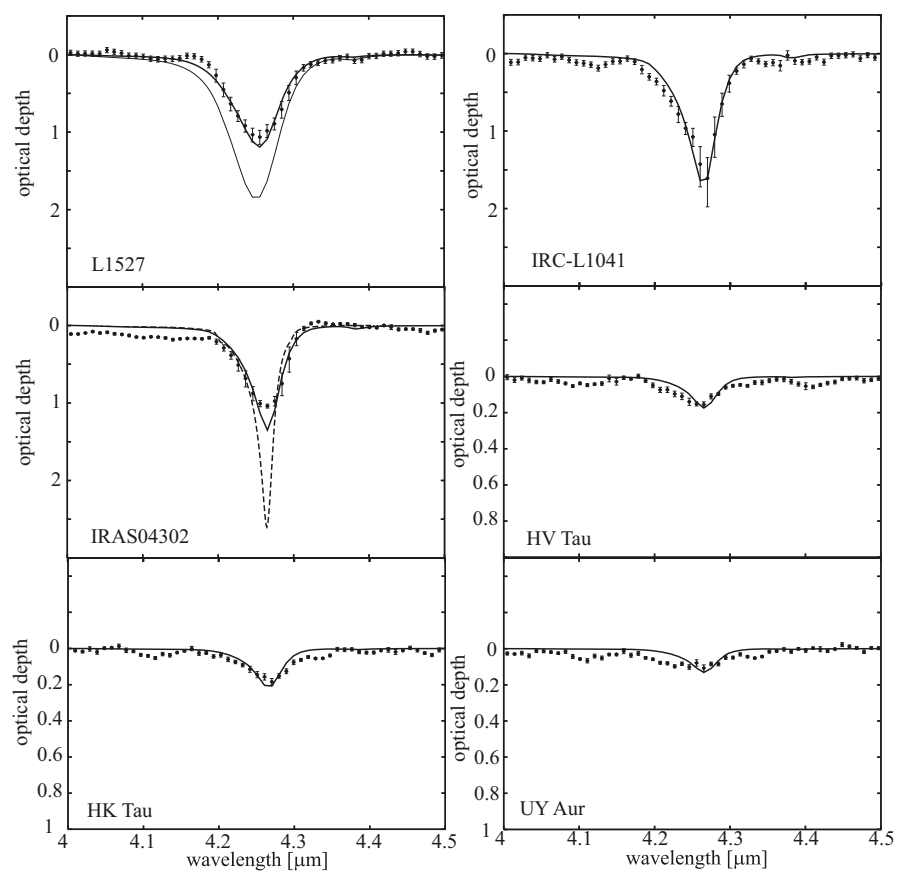

Fig. 6. Spectra fitted by the convolved laboratory spectrum of $\mathrm{CO}_{2}$ ice (solid line). The dashed lines depict the $\mathrm{CO}_{2}$ feature before convolution. In the panel of $\mathrm{L} 1527$, the $4.38 \mu \mathrm{m}$ feature is fitted by the convolved laboratory spectrum of ${ }^{13} \mathrm{CO}_{2}$ (thin solid line).

resolving power makes it difficult to distinguish between gas and ice. We tried to fit the observed feature with $\mathrm{CO}_{2}$ gas of constant temperature $(20 \mathrm{~K}, 70 \mathrm{~K}$, and $150 \mathrm{~K})$, but the observed features are better fitted by the pure $\mathrm{CO}_{2}$ ice.

We obtained the $\mathrm{CO}_{2}$ ice column densities listed in Table 2. The errors listed in Table 2 were derived from the errors in the absorption area. For class 0-I sources, the convolution function (the instrumental line profile or the $\mathrm{N} 3$ profile) might add errors to the column densities. For IRC-L1041, if we were to adopt the $\mathrm{N} 3$ profile, the $\mathrm{CO}_{2}$ column density should be larger by $\sim 30 \%$ than listed in Table 2; the wing region is reproduced better, but the agreement in the peak region is worse than in Fig. 6. For IRAS 04302, convolution with its N3 profile makes the absorption peak more flat and thus in closer agreement with the observation, while the $\mathrm{CO}_{2}$ column density is little changed.

We also detected $\mathrm{CO}_{2}$ absorption towards $\mathrm{HV}$ Tau and HK Tau. The absorption could, at least partially, originate in the circumstellar disks. The $\mathrm{H}_{2} \mathrm{O}$ absorption towards $\mathrm{HK}$ Tau could originate solely in the disk of HK Tau B (Sect. 4.2). If so, the detected $\mathrm{CO}_{2}$ ice towards $\mathrm{HK}$ Tau should also be in the edgeon disk, since it is unlikely that the foreground clouds contain $\mathrm{CO}_{2}$ but no $\mathrm{H}_{2} \mathrm{O}$. The $\mathrm{CO}_{2}$ absorptions of $\mathrm{HK}$ Tau and HV Tau are, however, deeper than $10 \mathrm{mJy}$, while the fluxes of HV Tau C and HK Tau B seem to reach a maximum in the $K$ band, and are smaller than $10 \mathrm{mJy}$ at $3.8 \mu \mathrm{m}$ and probably at longer wavelength (Sect. 4.2). Hence, we derived the $\mathrm{CO}_{2}$ ice column density by normalizing the spectrum with the continuum plotted in Fig. 2, as if the $\mathrm{CO}_{2}$ ice originates in the foreground clouds of the primary.

The relation between column densities of $\mathrm{CO}_{2}$ ice and $\mathrm{H}_{2} \mathrm{O}$ ice is plotted in Fig. 7. Our class 0-I stars show similar $\mathrm{CO}_{2} / \mathrm{H}_{2} \mathrm{O}$ ratios to those observed by Pontoppidan et al. (2008) towards embedded low-mass YSOs using SST. Towards HV Tau, HK Tau, and UY Aur, on the other hand, the column densities of $\mathrm{H}_{2} \mathrm{O}$ ice and $\mathrm{CO}_{2}$ ice are much lower than observed 
Y. Aikawa et al.: AKARI observations of ice absorption bands towards edge-on YSOs

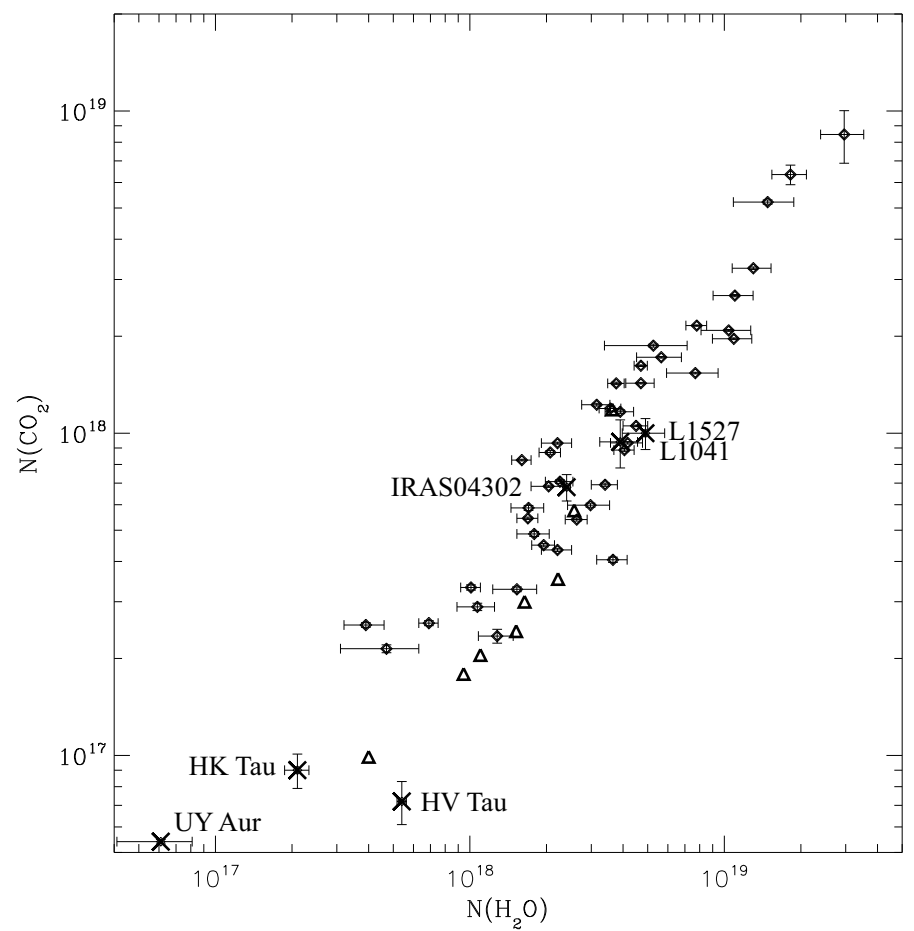

Fig. 7. The relation between column densities of $\mathrm{H}_{2} \mathrm{O}$ ice and $\mathrm{CO}_{2}$ ice towards our targets (crosses) plotted over Fig. 6 of Pontoppidan et al. (2008); the diamonds are low-mass stars observed by Pontoppidan et al. (2008), and the open triangles are the background stars from Whittet et al. (2007) and Knez et al. (2005).

by Pontoppidan et al. (2008), assuming that the detected absorption features all originate in the foreground clouds. The $\mathrm{CO}_{2} / \mathrm{H}_{2} \mathrm{O}$ ratios toward these class II objects, however, agree with the range of ratios for low-mass YSOs from Pontoppidan et al. (2008).

We note that the ${ }^{13} \mathrm{CO}_{2}$ band at $4.38 \mu \mathrm{m}$ is apparent in the spectra of L1527 and IRC-L1041-2. The laboratory $\mathrm{CO}_{2}$ profile used to fit the $4.27 \mu \mathrm{m}$ feature contains ${ }^{13} \mathrm{CO}_{2}$ with the terrestrial ratio ${ }^{12} \mathrm{C} /{ }^{13} \mathrm{C}=89$. In L1527, we fitted the $4.38 \mu \mathrm{m}$ feature (thin solid line in Fig. 6) with the laboratory spectrum. The absorption area $\left(\int \tau \mathrm{d} v\right)$ of the thin solid line is about two times larger than that of the thick solid line, indicating that the ${ }^{12} \mathrm{CO}_{2} /{ }^{13} \mathrm{CO}_{2}$ ratio could be lower than the terrestrial value. However, when considering the $\mathrm{S} / \mathrm{N}$, we found that the observed $4.38 \mu \mathrm{m}$ feature is consistent with the thick solid line in Fig. 6, which fits the $4.27 \mu \mathrm{m}$ feature; the terrestrial ratio ${ }^{12} \mathrm{C} /{ }^{13} \mathrm{C}=89$ are not significantly inconsistent with our observational data.

\section{6. $C O$ and $X C N$}

We found that $\mathrm{CO}$ ice has an absorption band at $4.67 \mu \mathrm{m}$. Figure 8 shows the spectra around this wavelength towards L1527 and IRC-L1041-2, IRAS 04302, HK Tau, and UY Aur.

Both L1527 and IRC-L1041-2 clearly have double-peaked profiles. The blue peak coincides with the XCN band, and the red peak matches the $\mathrm{CO}$ ice band. Although the double peak can be reproduced by combining XCN and CO ice, the observed features have an additional component at $\sim 4.7-4.75 \mu \mathrm{m}$. We assume that this corresponds to $\mathrm{CO}$ gas because, owing to the low sublimation temperature $(\sim 20 \mathrm{~K})$, CO can be more easily sublimated and excited near the protostar than $\mathrm{CO}_{2}$. With the low spectral resolution of IRC, gaseous $\mathrm{CO}$ vibrational bands cannot be observed as lines but become a broad double-peaked $(P$ branch and
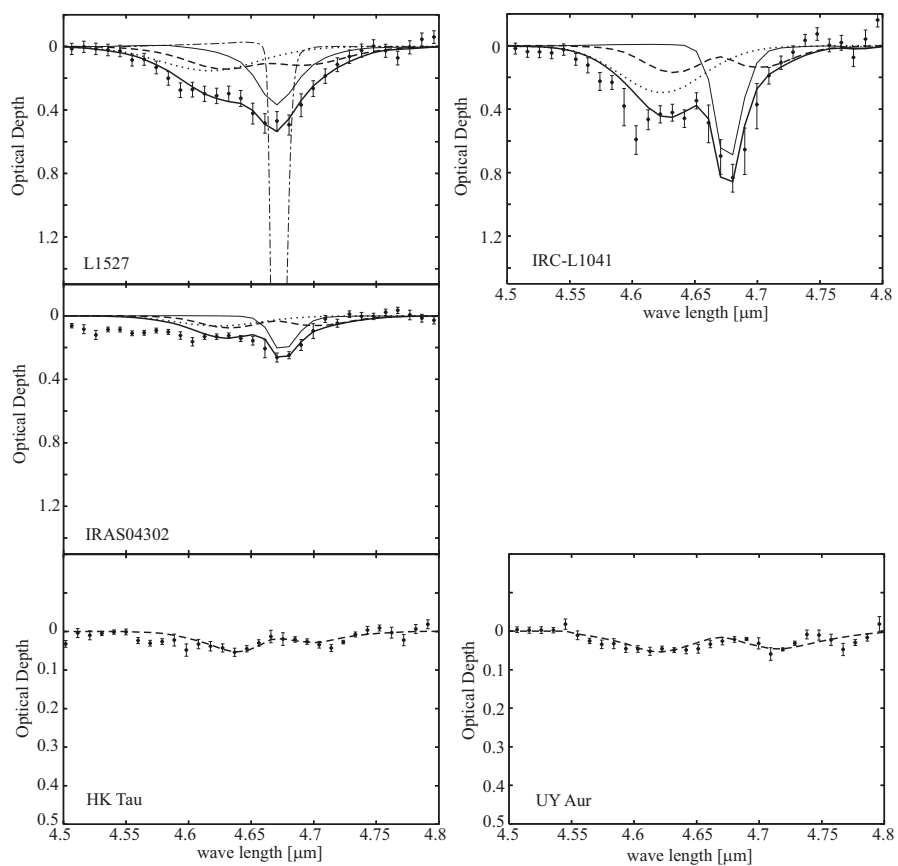

Fig. 8. The spectra around the $\mathrm{CO}$ ice absorption band fitted by a combination (thick solid line) of $\mathrm{CO}$ gas (dashed line), XCN feature (dotted line), and $\mathrm{CO}$ ice on the $\mathrm{CDE}$ grain model (thin solid line). The dotdashed line in the panel of L1527 depicts the CO ice feature before the convolution with the $\mathrm{N} 3$ profile.

$R$ branch) feature. It is thus difficult to derive the CO gas column density from our data. However, it is important to subtract the $\mathrm{CO}$ gas absorption, since the $R$ branch of the $\mathrm{CO}$ gas feature coincides with the XCN feature. The distance between the $P$ branch and $R$ branch increases with temperature. We fitted the absorption at $4.7-4.75 \mu \mathrm{m}$ with a $\mathrm{CO}$ gas profile at $70 \mathrm{~K}$ that had been smoothed to the low spectral resolution (dashed lines in Fig. 8).

After the subtraction of $\mathrm{CO}$ gas absorption, we fitted the $\mathrm{XCN}$ feature, assuming a Gaussian peaking at $4.62 \mu \mathrm{m}$ with FWHM of $29.1 \mathrm{~cm}^{-1}$ (Gibb et al. 2004) (dotted lines in Fig. 8), and the $\mathrm{CO}$ ice feature using the CDE grain model of Ehrenfreund et al. (1997) (thin solid lines in Fig. 8). The dotdashed line in Fig. 8 depicts the laboratory spectrum before convolution, and the thick solid lines in Fig. 8 shows the sum of the three fitted components.

The absorption feature around $4.67 \mu \mathrm{m}$ is also observed towards HK Tau and UY Aur, but the feature does not peak at $4.67 \mu \mathrm{m}$. We tentatively assigned the absorption to gaseous $\mathrm{CO}$ at $70 \mathrm{~K}$ and $150 \mathrm{~K}$ for HK Tau and UY Aur, respectively (dashed lines in Fig. 8).

Table 2 lists the estimated column densities of $\mathrm{CO}$ ice. The correlation between the column densities of $\mathrm{CO}$ ice and $\mathrm{H}_{2} \mathrm{O}$ ice is plotted in Fig. 9 over the correlation towards field stars of Whittet et al. (2007). The gray square depicts the ice column density ratio towards class I protostar Elias 29 (Boogert 2000). Our targets show higher $\mathrm{CO} / \mathrm{H}_{2} \mathrm{O}$ ratios than Elias 29, probably because our targets are edge-on objects. IRAS 04302 shows a lower ratio of $\mathrm{CO} / \mathrm{H}_{2} \mathrm{O}$ than field stars, indicating that its circumstellar material is warmer and/or less dense than typical molecular cloud gas.

Assuming that the $\mathrm{XCN}$ is $\mathrm{OCN}^{-}$, we adopt the band strength of $A(\mathrm{XCN})=5 \times 10^{-17} \mathrm{~cm} \mathrm{~mol}^{-1}$, and estimate the $\mathrm{OCN}^{-}$column density to be $(0.5-2.5) \times 10^{17} \mathrm{~cm}^{-2}$ towards 


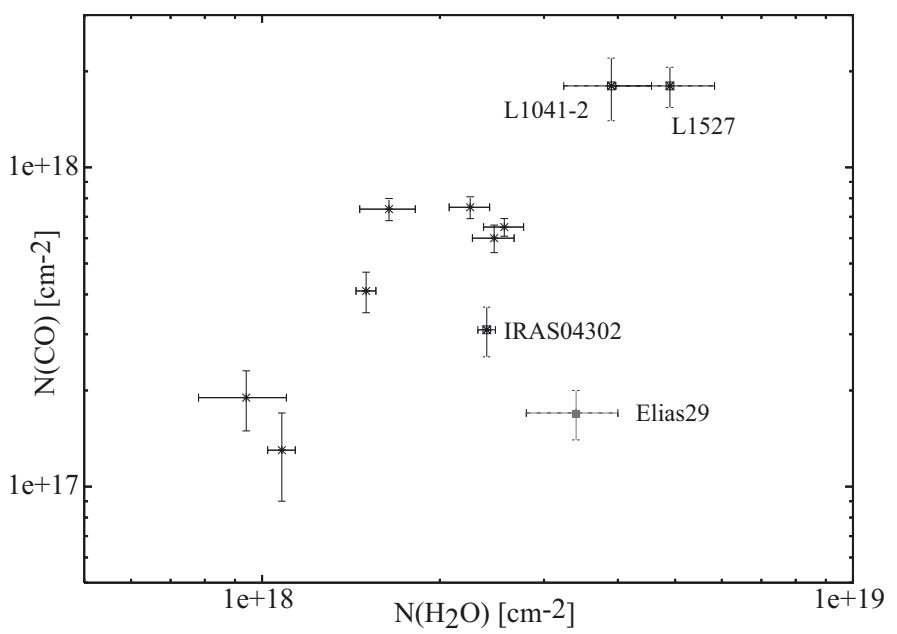

Fig. 9. The relation between the column densities of $\mathrm{H}_{2} \mathrm{O}$ ice and $\mathrm{CO}$ ice towards our targets (square). The crosses are ice columns towards field stars observed by Whittet et al. (2007). The gray square depicts the ice column density towards the low-mass class I protostar Elias29 (Boogert et al. 2000).

class 0-I objects (Table 2). The column density ratio of $\mathrm{OCN}^{-} / \mathrm{H}_{2} \mathrm{O}$ ranges from $2.2 \%$ (IRAS 04302 ) to $6.4 \%$ (L10412). Weintraub et al. (1994) detected the XCN feature towards a T Tauri star RNO 91 and derived an upper limit to the $\mathrm{XCN} / \mathrm{H}_{2} \mathrm{O}$ ratio of $\lesssim 9 \%$. More recent works, on the other hand, have derived much lower $\mathrm{XCN} / \mathrm{H}_{2} \mathrm{O}$ ratio towards low-mass YSOs. van Broekhuizen et al. (2005) observed low-mass YSOs with VLT; the column density ratio of $\mathrm{OCN}^{-} / \mathrm{H}_{2} \mathrm{O}$ is $\leq 0.85 \%$. Although CO gas can contribute to the absorption at $\sim 4.62 \mu \mathrm{m}$ in our spectra, its contribution is subtracted by referring to the absorption at $4.7-4.75 \mu \mathrm{m}$. In laboratory experiments, thermal or UV processing of $\mathrm{N}$-bearing ice produces $\mathrm{OCN}^{-}$(Schutte $\&$ Greenberg 1997). Since our targets are edge-on objects, our lines of sight may preferentially trace the surface region of the disks (or the dense torus region of the envelope) where $\mathrm{OCN}^{-}$ is produced by UV irradiation. The $\mathrm{OCN}^{-}$column densities towards IRC-L1041 and IRAS 04302, however, should be taken with caution, since there is residual absorption in the $4.5-4.6 \mu \mathrm{m}$ region (Fig. 8). If there are other unknown carriers at these wavelengths, the estimated $\mathrm{OCN}^{-}$column densities are upper limits.

Both L1527 and IRC-L1041-2 show weak absorption at $\sim 4.78 \mu \mathrm{m}$. Although it is close to the peak of ${ }^{13} \mathrm{CO}$ ice feature, the observed peak is at a slightly shorter wavelength than that of ${ }^{13} \mathrm{CO}$.

\subsection{OCS}

IRC-L1041-2 has an absorption feature at $\sim 4.9 \mu \mathrm{m}$. This coincides with the OCS ice band, which can be approximated by a Gaussian peaking at $4.91 \mu \mathrm{m}$ with a FWHM of $19.6 \mathrm{~cm}^{-1}$ (Gibb et al. 2004; Palumbo et al. 1997; Hudgins et al. 1993). Although the feature is at the edge of the observable wavelength range of the IRC and should be treated with caution, it does not disappear even if we change the combination of frames to be stacked. We fitted the spectrum with a Gaussian (Fig. 10). Assuming a band strength of $1.7 \times 10^{-18} \mathrm{~cm}$ molecule ${ }^{-1}$ (Hudgins et al. 1993), the OCS column density towards IRC-L1041-2 is $6.2 \times 10^{16} \mathrm{~cm}^{-2}$. If the abundance of $\mathrm{H}_{2} \mathrm{O}$ ice relative to hydrogen nuclei is $1 \times 10^{-4}$, the hydrogen column density in the line of sight is $3.9 \times 10^{22} \mathrm{~cm}^{-2}$, and the relative abundance of OCS ice to

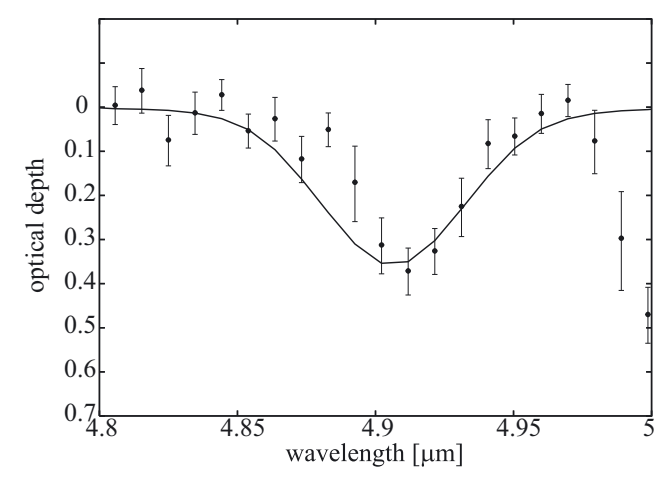

Fig. 10. Spectrum of IRC-L1041-2 at $4.9 \mu \mathrm{m}$. The solid line is a Gaussian with FWHM $19.6 \mathrm{~cm}^{-1}$ approximating the OCS absorption feature.

hydrogen is $1.6 \times 10^{-6}$, which is much higher than the abundance of gaseous OCS, $1 \times 10^{-9}$, observed towards TMC- 1 (Irvine et al. 1987).

\section{Discussion}

\subsection{Location of the lce}

Young stellar objects have a complicated structure: a protostar, circumstellar disk, envelope, and outflow cavity. Furthermore, some objects might be accompanied by either ambient or foreground clouds. In our observation of edge-on YSOs, the light source is not necessarily the central star. The NIR light of 3-5 $\mu \mathrm{m}$ can originate from, for example, a wall of the outflow cavity that scatters the stellar light. The light source can thus be spatially extended relative to the projected spatial scale of ice abundance structures, even if it looks like a point source (e.g. Tobin et al. 2008, 2010), and the absorbing material in the envelope and/or disk also has non-uniform spatial distributions. Although the radiative transfer modeling of such an envelope and disk system is beyond the scope of the present work, it is important to discuss the location of ice in our targets.

\subsubsection{Class $0-1$ objects}

Among the three class 0-I objects, L1527 is the most wellstudied. Its bolometric temperature is less than $70 \mathrm{~K}$, suggesting that the object is deeply embedded in dense gas (Furlan et al. 2008). Tobin et al. (2008) constructed an axisymmetric model of the envelope and disk with an outflow cavity referring to the SST images and SED of the object. According to the model, the "central object" we see in the N3 image is scattered light from the "inner cavity" with a size of $\sim 100$ AU. If so, the ice absorption bands in our spectrum most likely originate in the envelope rather than the disk. The envelope density ranges from about $10^{8} \mathrm{~cm}^{-3}$ to $10^{5} \mathrm{~cm}^{-3}$ at $\sim 100 \mathrm{AU}$ to $15000 \mathrm{AU}$. The integrated envelope column density in the line of sight is $\sim 1 \mathrm{~g} \mathrm{~cm}^{-2}$, which corresponds to a hydrogen column density of $N_{\mathrm{H}} \sim 4 \times 10^{23} \mathrm{~cm}^{-2}$. This would, however, be an overestimate, because the envelope structure is not axisymmetric in reality.

Owing to the similarity of the continuum spectra, we expect L1041 and L1527 to have a similar physical structure. Varying depths of ice absorptions among these objects would thus probe the difference in the ice composition in the envelope.

The SED modeling of the YSOs suggests that the envelope of IRAS 04302 is slightly less dense than L1527 (Furlan et al. 2008). We found smaller column densities of ices and lower 
$\mathrm{CO} / \mathrm{CO}_{2}$ ice ratios towards IRAS 04302 than L1527, which also indicates that IRAS 04302 is more evolved and has a less massive envelope.

Although our observations probe ice in envelopes rather than disks, the envelope material along our lines of sight (perpendicular to the rotation axis and outflow) is more likely to end up in the forming disks than be dissipated in the clouds. The high $\mathrm{CO} / \mathrm{CO}_{2}$ ice ratio indicates that the majority of gases in the lines of sight are still dense and cold. The $\mathrm{CO}_{2} / \mathrm{H}_{2} \mathrm{O}$ ratio towards our objects is similar to the ratio observed by Pontoppidan et al. (2008), who observed low-mass YSOs with various inclinations; the $\mathrm{CO}_{2} / \mathrm{H}_{2} \mathrm{O}$ ratio does not seem to vary with the inclination angle.

The $\mathrm{CO}$ gas absorption and deep absorption feature of $\mathrm{OCN}^{-}$, on the other hand, would originate in the warm $(T \geq$ $20 \mathrm{~K}$ ) and irradiated region near the protostar. However, other signs of heating by the protostar, such as crystalline feature of $\mathrm{H}_{2} \mathrm{O}$ ice (Schegerer \& Wolf 2010) and $\mathrm{CO}_{2}$ gas, are not found in our data. We may need higher spatial resolution and spectral resolution to detect these features.

\subsubsection{Edge-on class II objects: ASR41 and 2MASS J1628137}

We estimated the $\mathrm{H}_{2} \mathrm{O}$ ice column density from our observations to be $7.8 \times 10^{17} \mathrm{~cm}^{-2}$ towards ASR41 and $6.7 \times 10^{17} \mathrm{~cm}^{-2}$ towards 2MASS J1628137. These values are apparently much lower than we expect from the theoretical prediction of high $\mathrm{H}_{2} \mathrm{O}$ ice abundance $n\left(\mathrm{H}_{2} \mathrm{O}\right) / n_{\mathrm{H}} \sim 10^{-4}$ in the disk midplane. The shallow absorption is, however, caused by a geometric effect. Pontoppidan et al. (2005) predicts that the $3 \mu \mathrm{m}$ water band is the deepest for the disk inclination of $\sim 70^{\circ}$ and becomes shallower for higher inclination angles. If the inclination angle is $\geq 72^{\circ}$, the optical depth of the band is predicted to be $\sim 0.6$, which is comparable to our observation of ASR41. Since the light source (scattered stellar light) is more extended than the absorbing material, Eq. (2) underestimates the ice column density towards edge-on disks.

Possible contributions to the observed ice column densities from foreground clouds should also be considered. In the Taurus molecular cloud, the average $\mathrm{H}_{2} \mathrm{O}$ abundance is about $8.6 \times 10^{-5}$ relative to hydrogen nuclei at $A_{\mathrm{v}} \geq 3 \mathrm{mag}$ (Whittet et al. 1993; Chair et al. 2011). The $\mathrm{H}_{2} \mathrm{O}$ ice is not detected at lower $A_{\mathrm{v}}$, which indicates that $\mathrm{H}_{2} \mathrm{O}$ ice is easily destroyed by photolysis or photodesorption near the cloud surface. Hodapp et al. (2004) estimated that the density and size of the molecular cloud around ASR 41 are $2.0 \times 10^{-20} \mathrm{~g} \mathrm{~cm}^{-3}$ and $10^{4} \mathrm{AU}$, which corresponds to $A_{\mathrm{V}} \sim 1 \mathrm{mag}$. Although the relative abundance and the threshold $A_{\mathrm{v}}$ varies from cloud to cloud, the column density of the foreground gas towards ASR41, $A_{\mathrm{v}} \sim 1 \mathrm{mag}$, is too small to contribute significantly to the $\mathrm{H}_{2} \mathrm{O}$ ice absorption band. Therefore, the observed $\mathrm{H}_{2} \mathrm{O}$ ice most likely originates in the disk around ASR41.

The foreground visual extinction towards 2MASSJ 1628137 , on the other hand, is estimated to be $A_{\mathrm{V}}=2.1 \pm 2.6 \mathrm{mag}$ (Grosso et al. 2003 ). Assuming that the $\mathrm{H}_{2} \mathrm{O}$ ice abundance and threshold $A_{\mathrm{v}}$ are the same as in Taurus, the upper limit of the foreground $\mathrm{H}_{2} \mathrm{O}$ ice column density is $\sim 2 \times 10^{17} \mathrm{~cm}^{-2}$, which is smaller than the observed $\mathrm{H}_{2} \mathrm{O}$ ice column $\left(6.7 \times 10^{17} \mathrm{~cm}^{-2}\right)$. Hence, we conclude that most or all of the $\mathrm{H}_{2} \mathrm{O}$ ice observed towards 2MASSJ 1628137 originates in the disk.

\subsubsection{Other class II objects}

HV Tau and HK Tau are multiple systems. As the separation between the multiple components in these systems is smaller than the size of our PSF in the NIR, we cannot extract the spectra of the faint edge-on objects. Terada et al. (2007) observed $2-2.5 \mu \mathrm{m}$ and 3-4 $\mu \mathrm{m}$ spectra of these edge-on objects using Subaru and detected deep water absorption $(\tau \sim 1-1.5)$. The continuum flux of HV Tau $\mathrm{C}$ is $\sim 7.75 \mathrm{mJy}$ at $2.2 \mu \mathrm{m}$ and $\sim 7.14 \mathrm{mJy}$ at $3.8 \mu \mathrm{m}$, while the flux of HK Tau B is $\sim 15.9 \mathrm{mJy}$ at $2.2 \mu \mathrm{m}$ and $\sim 9.85 \mathrm{mJy}$ at $3.8 \mu \mathrm{m}$. Apparently, our spectra are dominated by the bright primaries. Both $\mathrm{H}_{2} \mathrm{O}$ and $\mathrm{CO}_{2}$ ice absorptions towards HV Tau should originate in both the disk and foreground clouds, since the depths of the absorption bands are larger than the continuum flux of the HV Tau C. Towards HK Tau, the depth of the $\mathrm{H}_{2} \mathrm{O}$ absorption is comparable to the continuum flux of $\mathrm{HK}$ Tau $\mathrm{B}$; if the $\mathrm{H}_{2} \mathrm{O}$ absorption originates in the disk around $\mathrm{HK}$ Tau $\mathrm{B}$, the $\mathrm{H}_{2} \mathrm{O}$ ice column density is $\sim 3 \times 10^{18} \mathrm{~cm}^{-2}$, which is comparable to the value obtained by Terada et al. (2007). The $\mathrm{CO}_{2}$ absorption, on the other hand, is deeper than the continuum flux of HK Tau B, and thus should originate at least partially in the foreground clouds. We also detected shallow $\mathrm{H}_{2} \mathrm{O}$ ice bands towards UY Aur, which again originate in the foreground component, since the inclination of the disk is $\sim 42$ degrees.

If the $\mathrm{H}_{2} \mathrm{O}$ absorptions towards $\mathrm{HV}$ Tau and HK Tau originated in the foreground clouds, the estimated $\mathrm{H}_{2} \mathrm{O}$ ice column densities would be smaller than the $\mathrm{H}_{2} \mathrm{O}$ ice column densities in the edge-on disks obtained by Terada et al. (2007) by about an order of magnitude; the $\mathrm{H}_{2} \mathrm{O}$ ice column density would be $3.31 \times 10^{18} \mathrm{~cm}^{-2}$ for HK Tau B, and (2.69-4.20) $\times 10^{18} \mathrm{~cm}^{-2}$ for HV Tau C. Our observations thus support the argument of Terada et al. (2007) that the disk component overwhelms the foreground component towards HK Tau B and HV Tau C.

\section{Conclusions}

We have observed ice absorption bands at 2.5-5 $\mu \mathrm{m}$ towards eight low-mass YSOs: three class 0-I protostellar cores with edge-on geometry, two edge-on class II objects, two multiple systems with edge-on class II, and one not-edge-on class II object.

Towards the class 0-I objects, L1527, IRC-L1041-2, and IRAS 04302, we have detected abundant $\mathrm{H}_{2} \mathrm{O}, \mathrm{CO}_{2}$, and $\mathrm{CO}$ ice in the envelope. The column density ratio of $\mathrm{CO}_{2}$ to $\mathrm{H}_{2} \mathrm{O}$ ice is $21-28 \%$, which coincides with the ratio observed by SST towards YSOs with various inclinations. The weak absorption at $\sim 4.1 \mu \mathrm{m}$ can be fitted by $\mathrm{HDO}$ ice; the $\mathrm{HDO} / \mathrm{H}_{2} \mathrm{O}$ ratio ranges from $2 \%$ to $22 \%$. The absorption in the vicinity of the $\mathrm{CO}$ band $(4.76 \mu \mathrm{m})$ is double-peaked and fitted by combining $\mathrm{CO}$ ice, $\mathrm{OCN}^{-}$, and $\mathrm{CO}$ gas. The large column density of $\mathrm{CO}$ ice suggests that the envelope is still very dense and cold, while $\mathrm{OCN}^{-}$ and $\mathrm{CO}$ gas features would originate in the region close to the protostar. The column density of $\mathrm{OCN}^{-}$is as high as $2-6 \%$ relative to $\mathrm{H}_{2} \mathrm{O}$, which is much higher than previous observations. Our lines of sight (high inclinations from the rotation axis) may preferentially trace the regions with high UV irradiation, such as the surface of a forming disk and/or torus envelope.

The spectrum of IRAS 04302 includes the $3.5 \mu \mathrm{m}$ absorption band, but the feature does not match either $\mathrm{CH}_{3} \mathrm{OH}$ or $\mathrm{CH}_{4}$. An OCS absorption band is tentatively detected towards IRCL1041-2.

Towards the edge-on class II objects, ASR41 and 2MASS J1628137-243139, we have detected the $\mathrm{H}_{2} \mathrm{O}$ band. The 
low optical depth of the water feature is due to geometrical effects (Pontoppidan et al. 2005). The detected water ice mainly originates in the disk.

Both HK Tau B and HV Tau C are edge-on class II objects in multiple systems. In our spectra, which are dominated by the primaries, we have detected the absorption of $\mathrm{H}_{2} \mathrm{O}$ ice and $\mathrm{CO}_{2}$ ice. Ices in both the edge-on disks and foreground clouds would contribute to the absorption, although $\mathrm{H}_{2} \mathrm{O}$ absorption towards HK Tau could originate solely in the disk. Even if the observed features are due to the foreground clouds, the $\mathrm{H}_{2} \mathrm{O}$ ice column densities in the clouds are much smaller than those observed towards HK Tau B and HV Tau C with Subaru (Terada et al. 2007), which confirms that the ice columns of the latter must originate in disks. The foreground $\mathrm{H}_{2} \mathrm{O}$ ice and $\mathrm{CO}_{2}$ ice are also detected towards UY Aur, which is not an edge-on system. We have tentatively detected CO gas towards HK Tau and UY Aur.

Acknowledgements. We would like to thank the project members of AKARI and AFSAS team for their help with the observation. We are grateful to Dr. D. Heinzeller for providing models of gas absorption features and to Prof. Whittet for his permission to use the $\mathrm{CO} / \mathrm{H}_{2} \mathrm{O}$ plot from Whittet et al. (2007). We would like to thank the anonymous referee for helpful comments that helped to improve the manuscript. This work was supported by a grant-in-aid for scientific research (19740103, 23540266, 23103004).

\section{References}

Aikawa, Y., Herbst, E., Roberts, H., \& Caselli, P. 2005, ApJ, 620, 330 Aikawa, Y., Wakelam, V., Garrod, T. R., \& Herbst, E. 2008, ApJ, 674, 984 André, P., Ward-Thompson, D., \& Barsony, M. 2000, Protostars and Planets IV, 59

Boogert, A. C. A., Tielens, A. G. G. M., Ceccarelli, C., et al. 2000, A\&A, 360, 683

Boogert, A. C. A., Blake, G. A., \& Tielens, A. G. G. M. 2002, ApJ, 577, 271

Boogert, A. C. A., Blake, G. A., \& Öberg, K. 2004, ApJ, 615, 344

Boogert, A. C. A., Pontoppidan, K. M., Knez, C., et al. 2008, ApJ, 678, 985

Boogert, A. C. A., Huard, T. L., Cook, A. M., et al. 2011, ApJ, 729, 92

Brooke, T. Y., Sellgren, K., \& Smith, R. G. 1996, ApJ, 459, 2009

Chair, J. E., Pendleton, Y. J., Allamandola, L. J., et al. 2011, ApJ, 731, 9

Dartois, E., Thi, W. F., Geballe, T. R., et al. 2003, A\&A, 399, 1020

Ehrenfreund, P., Boogert, A. C. A., Gerakines, P. A., Tielens, A. G. G. M., \& van Dishoeck, E. F. 1997, A\&A, 328, 649

Furlan, E., McClure, M.,Calvet, N., et al. 2008, ApJS, 176, 184

Geers, V. C., van Dishoeck, E. F., Visser, R., et al. 2007, A\&A, 476, 279

Gerakines, P. A., Schutte, W. A., Greenberg, J. M., \& van Dishoeck, E. F. 1995, A\&A, 296, 810

Gerakines, P. A., Schutte, W. A., \& Ehrenfreund, P. 1996, A\&A, 312, 289

Gibb, E. L., Whittet, D. C. B., Schutte, W. A., et al. 2000, ApJ, 536, 347

Gibb, E. L., Whittet, D. C. B., Boogert, A. C. A., \& Tielens, A. G. G. M. 2004, ApJS, 151, 35

Grim, R. J. A., Baas, F., Geballe, T. R., Greenberg, G. M., \& Schutte, W. 1991, A\&A, 243, 473
Grosso, N., Alves, J., Wood, K., et al. 2003, ApJ, 586, 296

Hioki, T., Itoh, Y., Oasa, Y., et al. 2007, ApJ, 134, 880

Hodapp, K. W., Walker, C. H., Reipurth, B., et al. 2004, ApJ, 601, L79

Honda, M., Inoue, A. K., Fukagawa, M., et al. ApJ, 690, L110

Hudgins, D. M., Sandford, S. A., Allamandola, L. J., \& Tielens, A. G. G. M. 1993, ApJS, 86, 713

Irvine, W. M., Goldsmith, P. F., \& Hjalmarson, A. 1987, in Interstellar processes, ed. D. J. Hollenbach, \& H. A. Jr. Thronson, 561

Knez, C., Boogert, A. C. A., Pontoppidan, K. M., et al. 2005, ApJ, 635, L145

Lacy, J. H., Carr, J. S., Evans, N. J.II, et al. 1991, ApJ, 376, 556

Léger, A., Gauthier, S., Defourneau, D., \& Rouan, D. 1983, A\&A, 117, 164

Monin, J.-L., \& Bouvier, J. 2000, A\&A, 356, L75

Murakami, H., Baba, H., Barthel, P., et al. 2007, PASJ, 59, 369

Murakawa, K., Tamura, M., \& Nagata, T. 2000, ApJS, 128, 603

Öberg, K. I., Boogert, A. C. A., Pontoppidan, K. M., et al. 2008, ApJ, 678, 1032

Ohyama, Y., Onaka, T., Matsuhara, H., et al. 2007, PASJ, 59, 411

Onaka, T., Matsuhara, H., Wada, T., et al. 2007, PASJ, 59, 401

Palumbo, M. E., Geballe, T. R., \& Tielens, A. G. G. M. 1997, ApJ, 479, 839

Parise, B., Simon, T., Caux, E., et al. 2003, A\&A, 410, 897

Parise, B., Caux, E., Castets, A., et al. 2005, A\&A, 431, 547

Pontoppidan, K. M., \& Dullemond, C. P. 2005, A\&A, 435, 595

Pontoppidan, K. M., Fraser, H. J., Dartois, E., et al. 2003a, A\&A, 408, 981

Pontoppidan, K. M., Dartois, E., van Dishoeck, E. F., Thi, W.-F., \& d'Hendecourt, L. 2003b, A\&A, 404, L17

Pontoppidan, K. M., Boogert, A. C. C., Fraser, H. J., et al. 2008, ApJ, 678, 1005

Sakai, N., Sakai, T., Hirota, T., \& Yamamoto, S. 2008, ApJ, 672, 371

Sakon, I., Onaka, T., Wada, T., et al. 2007, PASJ, 59, 483

Schutte, W. A., \& Greenberg, J. M. 1997, A\&A, 317, L43

Schegerer, A. A., \& Wolf, S. 2010, A\&A, 517, A87

Simon, M., Holfeltz, S. T., \& Taff, L. G. 1998, ApJ, 469, 890

Smith, R. G., Sellgren, K., \& Tokunaga, A. T. 1989, ApJ, 344, 413

Stapelfeldt, K. R., Krist, J. E., Menard, F., et al. 1998, ApJ, 502, L65

Terada, H., Tokunaga, A. T., Kobayashi, N., et al. 2007, ApJ, 667, 303

Teixeira, T. C., \& Emerson, J. P. 1999, A\&A, 351, 303

Thi, W. F., Pontoppidan, K. M., van Dishoeck, E. F., Dartois, E. \& d'Hendecourt, L. 2002, A\&A, 394, L27

Tobin, J. J., Hartmann, L., Calvet, N., \& D’Alessio, P. 2008, ApJ, 679, 1364

Tobin, J. J., Hartmann, L., Looney, L. W., \& Chiang, H.-F. 2010, ApJ, 712, 1010

van Broekhuizen, F. A., Keane, J. V., \& Schutte, W. A. 2004, A\&A, 415, 425

van Broekhuizen, F. A., Pontoppidan, K. M., Fraser, H. J., \& van Dishoeck, E. F. 2005, A\&A, 441, 249

van Broekhuizen, F. A., Groot, I. M. N., Fraser, H. J., van Dishoeck, E. F., \& Schlemmer, S. 2006, A\&A, 451, 273

van Dishoeck, E. F., Helmich, F. P., de Graauw, Th., et al. 1996, A\&A, 315, L349

Visser, R., van Dishoeck, E. F., Doty, S. D., \& Dullemond, C. P. 2009, A\&A, 495,881

Weintraub, D. A., Tegler, S. C., Kastner, J. H., \& Rettig, T. 1994, ApJ, 423, 674

Whittet, D. C. B. 1993, Dust and Chemistry in Astronomy (Bristol and Philadelphia: Institute of Physics Publishing), 9

Whittet, D. C. B., Gerakines, P. A., Tielens, A. G. G. M., et al. 1998, ApJ, 498, 159

Whittet, D. C. B., Shenoy, S. S., Bergin, E. A., et al. 2007, ApJ, 655, 332

Woitas, J., \& Leinert, Ch. 1998, A\&A, 338, 122

Wolf, S., Padgett, D., \& Stapelfeldt, K. R. 2003, ApJ, 588, 373

Zasowski, G., Kemper, F., Watson, D. M., et al. 2009, ApJ, 694, 459 